\title{
Localization via Visible Light Systems
}

\author{
This paper considers the problem of localization in visible light systems and \\ discusses localization techniques, cooperative architectures, iterative algorithms, \\ and optimal power allocation.
}

By Musa Furkan Keskin, Ahmet Dundar Sezer, and Sinan Gezici ${ }^{\circledR}$, Senior Member IEeE

ABSTRACT | Visible light communication (VLC) is an emerging paradigm that enables multiple functionalities to be accomplished concurrently, including illumination, highspeed data communications, and localization. Based on the VLC technology, visible light positioning (VLP) systems aim to estimate locations of VLC receivers by utilizing light-emitting diode (LED) transmitters at known locations. VLP presents a viable alternative to radio frequency (RF)-based positioning systems by providing inexpensive and accurate localization services. In this paper, we consider the problem of localization in visible light systems and provide an extensive survey of various location estimation techniques, accompanied by discussions of their relative merits and demerits within the context of accuracy and computational complexity. In addition, we investigate a cooperative VLP system architecture in which VLC receiver units are able to communicate with each other for the purpose of cooperation, and present a low-complexity, iterative localization algorithm to demonstrate the benefits of cooperation in VLP systems. Finally, we investigate optimal strategies for power allocation among LED transmitters to maximize the localization accuracy subject to power and illumination constraints.

KEYWORDS I Cooperation; direct positioning; localization; parameter estimation; power allocation; two-step positioning; visible light communication (VLC); visible light positioning (VLP)

\section{INTRODUCTION}

Visible light positioning (VLP) systems have recently attracted great attention due to their significant capabilities in localization related applications, especially in indoor scenarios [1], [2]. In indoor environments, VLP systems, combined with the

Manuscript received December 3, 2017; revised February 2, 2018; accepted March 27, 2018. Date of publication May 11, 2018; date of current version May 24, 2018. (Corresponding author: Sinan Gezici.)

The authors are with the Department of Electrical and Electronics Engineering, Bilkent University, 06800 Ankara, Turkey (e-mail: gezici@ee.bilkent.edu.tr).

Digital Object Identifier: 10.1109/JPROC.2018.2823500 power of the light-emitting diode (LED) technology, facilitate precise and accurate localization and provide low-cost solutions for applications that require compact and effective positioning systems. Due to the emerging developments in the LED technology, LEDs can also be used for communication and localization purposes besides their primary function of providing illumination [3]-[5]. The attractive features of LEDs such as long lifespan and low-power consumption make the LED technology the main ingredient in a reliable, robust, and efficient illumination system. In addition to these properties, LEDs have the capability of switching to different intensity levels at very fast rates; that is, they can be modulated at frequencies as high as $300 \mathrm{MHz}$ (much higher than the conventional lighting systems) and can be used to transmit data without causing visible flicker [6], [7]. Hence, LEDs can effectively serve multiple purposes of communication and localization as well as illumination.

\section{A. Features of Visible Light Systems}

In the context of communication and localization, visible light systems benefit from the desirable properties of the visible light and its spectrum. Compared to traditional wireless systems which employ the cluttered, scarce, and expensive radio frequency (RF) spectrum, VLP systems utilize the visible light portion of the electromagnetic spectrum, which is unlicensed and unregulated [4]. The visible light spectrum with its huge license free bandwidth facilitates high speed data transmission and reduces the costs for operators. Besides VLP systems, visible light communication (VLC) systems complement the RF-based communication systems and meet the highcapacity traffic demand for wireless networks by operating in the visible light spectrum [3], [6], [8].

In addition to its untapped spectrum, the LED-based visible light technology provides some advantages for both VLP and VLC systems in terms of reliability, robustness, and security [5], [9]. Compared to the transmitters in 
conventional RF-based systems, the power consumption of LEDs can be significantly lower. Since LEDs are already used for illumination purposes in indoor environments, they can be incorporated into VLP and VLC solutions without causing a substantial increase in the power consumption. In addition, the inherent line-of-sight (LOS) property of LEDs provides a secure data transmission for systems in which the transmitter and the receiver directly communicate via high-frequency visible light channel in a confined space by ensuring LOS clearance between them. The fundamental reason behind that feature is the inability of visible light to penetrate through opaque objects such as walls [10]. That characteristic of the visible light prevents intercell interference issues mostly encountered in RF-based systems and leads to efficient cell-based communication and localization systems separated by partitions such as walls [10]. Furthermore, visible light signals do not interfere with the RF signals in sensitive electronic devices and consequently they can freely be employed in applications where RF interference may cause issues for proper functioning of devices.

Although global positioning system (GPS) is commonly employed in many applications, it suffers from signal blockage and multipath propagation, which results in poor performance in indoor environments [11]. For that reason, other RF-based positioning systems are proposed in the literature for indoor applications [12]-[15]. However, those systems can easily be exposed to multipath interference and consequently their localization performance can degrade as the radio signals reaching a receiver via different multiple paths (e.g., due to reflections from objects such as walls) complicate the process of estimating location related parameters from the received signal. On the other hand, due to the nature of visible light channels, multipath effects are not as significant in VLP systems as those in RF-based positioning [8], [16]-[21]. Moreover, the widespread use of LEDs for illumination constitutes an opportunity to design a ubiquitous and economical positioning system, which functions efficiently in indoor environments [22]. In particular, LED-based VLP systems can easily be integrated into the existing lighting infrastructure (i.e., facilitates the reuse of existing infrastructure) for the purpose of localization in addition to its essential function of illumination without usually requiring rewiring. It is also important to note that VLP systems can be deployed not only for indoor applications but also for outdoor applications such as street lights, stop lights, and airport taxi-way lighting [23], [24]. In general, VLP systems can appropriately be employed in any application where LEDs are utilized.

\section{B. VLP Applications and Design Constraints}

VLP systems are used in a broad range of applications including automated vehicles, location-aware services, and asset tracking [1], [23], [25]-[27]. The VLP system employed in each application needs to satisfy different requirements in terms of various criteria such as cost, reliability, and robustness. As an example, a VLP system can be installed in a museum and with the help of such a system, the visitors of that museum can get information about an exhibit (e.g., a historical artifact) when they are in front of it. A handheld device (e.g., a smartphone) of a visitor can receive the signals transmitted by the LEDs placed near the exhibit and estimate the current position of the visitor. Then, it decides which exhibit the visitor is interested in and informs the visitor about the corresponding exhibit. Such an application may not have a very strict accuracy requirement for localization. However, there also exist applications in which highly accurate and precise positioning is required; e.g., robot navigation [28]. In order to estimate the position accurately and precisely, those applications require more advanced algorithms than those utilized in the museum application, and employ various techniques to enhance localization performance of VLP systems.

The design of VLP systems depends also on some crucial factors and constraints arising from the primal illumination purpose of LEDs. The primary function of the LEDs is to provide energy-efficient and high-quality illumination. For that reason, any VLP system designed based on LEDs should be suitable for that purpose and allow the LEDs to operate flawlessly without any restraints. Also, the signals transmitted by the LEDs in a VLP system should not cause any visible flicker, and the color changes in the LEDs should not be detectable by the human eye during signal transmission. In addition, a practical VLP system should satisfy lighting level requirements of an illumination system in which dimming control is a required feature. Last, it is worth noting that the relationship between the applied current and the light output of an LED is linear only in a limited dynamic range. Therefore, VLP systems should be designed in consideration of nonlinear characteristics of LEDs, as well [29]-[32].

\section{Localization Techniques in VLP Systems}

Similar to RF-based positioning systems [33]-[39], VLP systems consist mainly of two components, namely, transmitters and receivers. LEDs correspond to the transmitter part of the VLP system and send the necessary information (e.g., a position signal or a code) in order for the receiver to determine its own position in the system. On the receiving side of the VLP system, two different types of receivers can be employed in general [40]-[42], namely, photo detector (PD) and imaging sensor. In both cases, the received signals (i.e., lights) coming from the LEDs are used to estimate the localization parameters such as the relative distance and/ or direction of the LED transmitters. Then, the information gathered from the received signals is analyzed based on the positioning techniques to figure out the locations of the receivers. Regarding the types of receivers, the PD is a low-cost solution for the receiver part of the VLP system and provides energy-efficient and high-rate data communication. On the contrary, imaging sensors are often more costly and suitable for applications with low data rate requirements [43]. Recently, with improved cost-performance tradeoffs induced by new complementary metaloxide-semiconductor (CMOS) technologies, those sensors 
can already be found in smart devices such as smartphones and can readily be employed in desired applications [43].

In a VLP system, various approaches can be employed for location estimation, which can mainly be classified into two groups: direct positioning and two-step positioning. In the direct positioning approach, all the available information gathered from the received signals is directly exploited to estimate the position of the receiver without examining position related parameters in advance. In other words, this approach does not include any intermediate steps for parameter estimation and can provide the optimal solution of the localization problem. On the other hand, the two-step method performs position estimation in two separate stages. In the first stage, position related parameters are extracted, which is followed by estimation of the receiver position in the second stage via various algorithms and methods based on those parameters. The two-step positioning approach has a lower complexity than the direct positioning approach (which requires high data storage and communication capacity); however, it leads to a suboptimal solution as it does not use the received signals directly.

Within the context of two-step positioning, the studies in the literature consider different position related parameters such as received signal strength (RSS) [40], [44]-[46], time of arrival (TOA) [47]-[49], time difference of arrival (TDOA) [50]-[52], and angle of arrival (AOA) [53]-[56]. In VLP systems, RSS is a common parameter which is employed to gather information related to the distance and orientation of the VLC receiver with respect to the LED transmitter. Compared to the time-based parameters such as TOA and TDOA, the RSS parameter can be estimated in a low complexity manner since synchronization is not needed. On the contrary, the VLC receiver must be synchronized with each of the LED transmitters in order to estimate the distances between itself and the LED transmitters based on TOA measurements. Regarding the TDOA parameter, there is no need for synchronization between the VLC receiver and the LED transmitters but the LED transmitters must be synchronized among themselves so that the VLC receiver can estimate the distance difference between itself and each pair of LED transmitters based on the corresponding TDOA measurement. Last, AOA is a promising parameter for VLP systems and can efficiently be employed in the localization process. Based on the direction of the received signal, the AOA-based systems can adequately perform localization with the help of LOS connections between the LED transmitters and the VLC receiver.

Although the same types of position related parameters are employed in both VLP- and RF-based localization systems, the information carried by these parameters can be quite different. In particular, the RSS parameter employed in VLP systems has significantly higher accuracy than that in RF-based systems [1]. The main reason for this is related to the severity of multipath effects in RF-based systems. Namely, RF-based systems suffer from multipath interference and typically do not have an LOS path between the transmitter and the receiver. On the contrary, multipath effects are not as crucial in VLP systems as those in RF-based ones since VLP systems commonly have LOS paths between LED transmitters and VLC receivers and the diffuse components arising from multipath scattering are much weaker than the LOS component. Similar to the RSS parameter, the AOA parameter, which is measured based on received power levels at PDs, can also provide high accuracy in VLP systems compared to RF-based solutions for the aforementioned reasons [1]. In addition, it is important to note that RSS and AOA parameters provide a low-cost solution for VLP systems to estimate the position of a VLC receiver accurately since they do not require synchronization among VLC units. On the other hand, estimation of TOA and TDOA parameters requires precise synchronization and highly accurate time measurements, thus rendering these metrics relatively costly in practical applications.

After the position related parameters are obtained in the first step of the two-step positioning approach, a VLP system can apply numerous algorithms and techniques in the second step to finalize the localization process. The algorithms and techniques employed to that aim in the literature can be grouped as follows. First, the proximity-based methods perform localization based on the data obtained from the nearest LED and suits for applications in which very accurate position information is not required [57], [58]. Second, the geometric methods determine the position of the VLC receiver by analyzing the position related parameters such as TOA and AOA in a geometric fashion [59], [60]. In other words, the extracted information in the first step is evaluated in the second step with the help of some geometric processes such as trilateration and triangulation. The statistical methods, which constitute the third group, benefit from the statistical distributions of the parameters obtained in the first step and derive the position estimators in view of those statistical properties [55], [61]-[64]. Last, in the fingerprinting methods, the estimated parameters based on the online measurements performed by the VLC receiver are compared with the data in a previously obtained database and a matching algorithm decides the position of the VLC receiver in the system [65], [66].

Apart from the algorithms and techniques that can be applied in the second step of the two-step positioning scheme, alternative approaches can also be employed to improve the localization performance of VLP systems [67]-[69]. As in RF-based positioning systems [34], [70]-[72], cooperation among the entities in a VLP system can enhance the accuracy of localization [67], [68]. Moreover, the optimal power allocation approaches can be designed for LED transmitters to enhance the localization accuracy of the VLP system [69]. In these approaches, the powers of the LEDs in the system can be set to the optimal levels instead of operating all the LEDs at the same power level while taking power and illumination constraints into account during the design of the system. 


\section{Summary and Organization}

In general, the aim of this paper is to provide an overview of the position estimation methods for VLP systems. The main points presented in the paper can be summarized as follows.

- We present the state-of-the-art methods for position estimation in visible light systems, which can essentially be classified as direct and two-step approaches.

- We investigate different types of position related parameters employed in VLP systems, such as RSS, TOA, TDOA, and AOA, and focus on positioning techniques that utilize those parameters.

- We discuss the effects of cooperation on the performance of VLP systems and present an iterativegradient-projection-based cooperative localization algorithm, motivated by a quasi-convex feasibility approach.

- We study the problem of optimal power allocation among LED transmitters to maximize the localization performance under practical constraints (e.g., illumination) and illustrate the resulting accuracy improvements.

The remainder of the paper is organized as follows. Section II presents position estimation methods for VLP systems. In Section III, positioning techniques are discussed in the presence of cooperation among the entities in a VLP system. Section IV investigates the optimal power allocation problem for LEDs in a VLP system in consideration of illumination constraints, and then provides numerical examples for the solutions of the optimization problems. Finally, Section $\mathrm{V}$ concludes the paper and addresses some possible directions for future work.

\section{POSITION ESTIMATION METHODS}

In this section, we discuss various positioning schemes for VLP systems to present a comprehensive insight on the state-of-the-art techniques for parameter extraction and position estimation (see Fig. 1). In both RF- and VLC-based systems, position estimation is performed by exchanging signals between nodes with known locations (called anchor/reference nodes) and nodes whose locations are to be estimated (called target/agent nodes) [37], [61]. (Also, signal exchanges among target nodes can provide additional location information, which is utilized in cooperative positioning systems; see Section III.) For VLP systems, LED transmitters, which have known locations and are typically attached to the ceiling of a room in indoor scenarios, function as anchor nodes, and VLC receivers, equipped with PDs, seek to determine their own locations based on signals transmitted by LEDs and detected through PDs. ${ }^{1}$ Hence, VLC receivers commonly perform self-localization [81] by utilizing incoming VLC signals and known locations of LEDs that emit those signals. In the following, we focus on the two well-known positioning paradigms, namely, direct positioning and two-step positioning. Direct positioning consists of a single step for location estimation while twostep positioning is conducted in two separate phases [61], [63], [82]-[84], as detailed in the following sections.

\section{A. Direct Positioning}

In the direct positioning approach, localization relies on a single-step estimation procedure that utilizes the entire received waveforms to infer the location of a target node [61], [81], [82], [85]. As opposed to the conventional twostep approach, the direct positioning technique exploits the whole received signals to estimate the location without intermediate steps for extracting location-dependent parameters [61], [82]. The direct positioning method has successfully been applied to both RF- [82]-[88] and VLCbased [63], [89] localization systems. To investigate the

${ }^{1}$ In this paper, PD-based VLC receivers are considered for VLP systems. For imaging-sensor-based VLP systems, see [41], [42], and [73][76]. The reader is referred to [25], [26], [46], and [77]-[80] for some practical implementations of VLP systems.

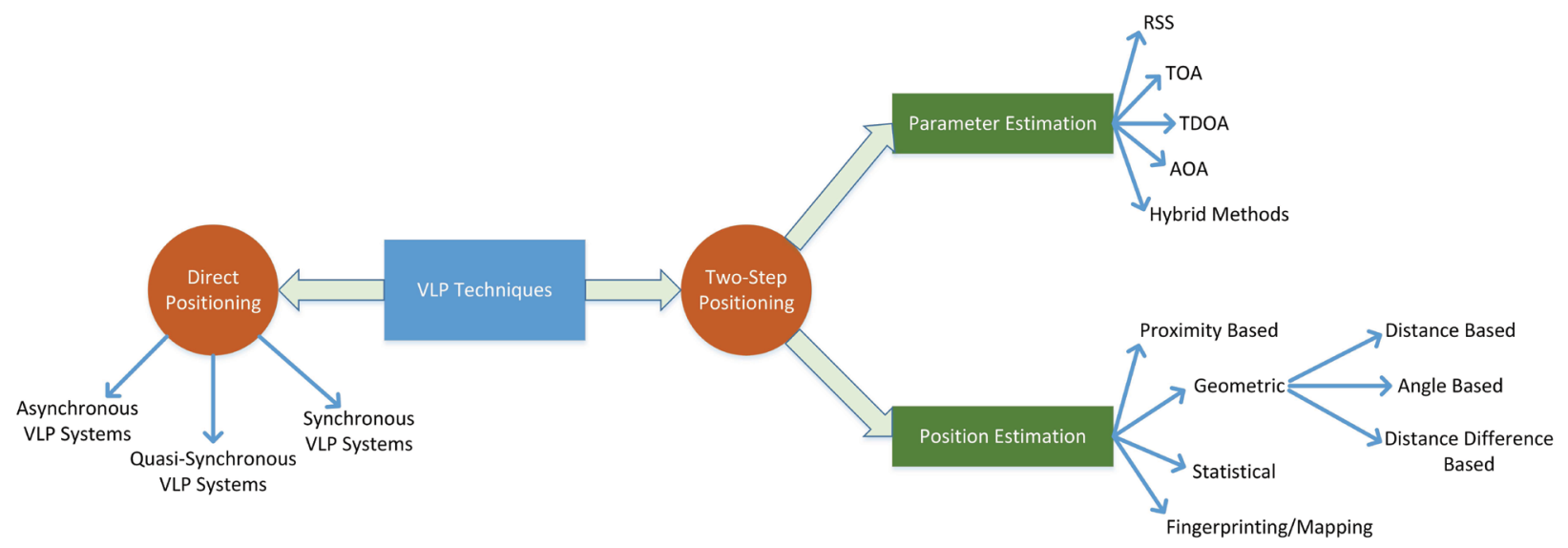

Fig. 1. Classification of localization techniques for VLP systems. 
direct positioning approach in VLP systems, we describe the signal model at a VLC receiver, present the direct-positioning-based maximum-likelihood (ML) estimators, and provide performance limits for localization in the following sections.

1) Received Signal Model: Consider a VLP system with $N_{\mathrm{L}}$ LED transmitters and a VLC receiver. Assuming a LOS scenario between each LED transmitter and the VLC receiver [1], [47], the received signal at the PD of the VLC receiver due to the ith LED transmitter can be expressed as [47]

$$
r_{i}(t)=\alpha_{i} R_{p} s_{i}\left(t-\tau_{i}\right)+\eta_{i}(t)
$$

for $i \in\left\{1, \ldots, N_{\mathrm{L}}\right\}$ and $t \in\left[T_{1, i}, T_{2, i}\right]$, where $T_{1, i}$ and $T_{2, i}$ determine the observation interval for the signal emitted by the $i$ th LED transmitter, $\alpha_{i}$ is the optical channel attenuation between the ith LED transmitter and the VLC receiver $\left(\alpha_{i}>0\right), R_{p}$ denotes the responsivity of the $\mathrm{PD}, s_{i}(t)$ is the transmitted signal of the ith LED transmitter, which is nonzero over an interval of $\left[0, T_{s, i}\right], \tau_{i}$ is the TOA of the signal emitted by the ith LED transmitter at the VLC receiver, and $\eta_{i}(t)$ is zero-mean additive white Gaussian noise with spectral density level $\sigma^{2}$. To facilitate independent processing of signals coming from different LED transmitters, a type of multiple-access scheme, such as frequency-division or timedivision multiple access [90], [91], can be employed. Hence, the signals corresponding to different LED transmitters do not interfere with each other at the VLC receiver, and the noise processes $\eta_{1}(t), \ldots, \eta_{N_{\mathrm{L}}}(t)$ become independent.

Let $\boldsymbol{l}_{\mathrm{r}}=\left[\begin{array}{lll}l_{\mathrm{r}, 1} & l_{\mathrm{r}, 2} & l_{\mathrm{r}, 3}\end{array}\right]^{T}$ and $\boldsymbol{l}_{\mathrm{t}}^{\mathrm{i}}=\left[\begin{array}{lll}l_{\mathrm{t}, 1}^{\mathrm{i}} & l_{\mathrm{t}, 2}^{\mathrm{i}} & l_{\mathrm{t}, 3}^{\mathrm{i}}\end{array}\right]^{T}$ denote, respectively, the locations of the VLC receiver and the $i$ th LED transmitter, and $\left\|\boldsymbol{l}_{\mathrm{r}}-\boldsymbol{l}_{\mathrm{t}}^{\mathrm{i}}\right\|$ represent the distance between the ith LED transmitter and the VLC receiver. Then, the TOA parameter in (1) can be modeled as

$$
\tau_{i}=\frac{\left\|\boldsymbol{l}_{\mathrm{r}}-\boldsymbol{l}_{\mathrm{t}}^{\mathrm{t}}\right\|}{c}+\Delta_{i}
$$

where $c$ is the speed of light, and $\Delta_{i}$ denotes the time offset between the clocks of the ith LED transmitter and the VLC receiver. In synchronous VLP systems, where all the LED transmitters and the VLC receiver are synchronized to a common clock, $\Delta_{i}=0$ for $i=1, \ldots, N_{\mathrm{L}}$. In asynchronous systems, synchronization exists neither among the LED transmitters nor between the LED transmitters and the VLC receiver, in which case $\Delta_{i}$ 's can be modeled as deterministic unknown parameters. Finally, for quasi-synchronous VLP systems [92], where the LED transmitters are synchronized to a common time reference but are not synchronized with the VLC receiver, $\Delta_{i}=\Delta$ for $i=1, \ldots, N_{\mathrm{L}}$.

Based on the Lambertian model [93], the optical channel attenuation $\alpha_{i}$ in (1) can be expressed as

$$
\alpha_{i}=\frac{\left(m_{i}+1\right) A_{\mathrm{R}} \cos ^{m_{i}}\left(\phi_{i}\right) \cos \left(\theta_{i}\right)}{2 \pi\left\|\boldsymbol{l}_{\mathrm{r}}-\boldsymbol{l}_{\mathrm{t}}^{i}\right\|^{2}}
$$

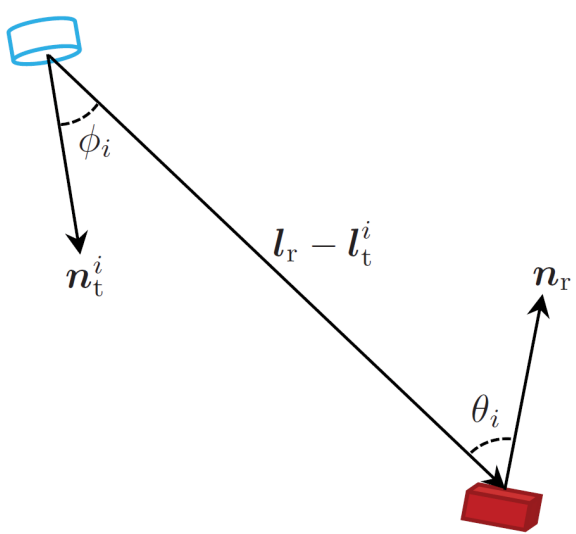

Fig. 2. Illustration of configuration parameters in the Lambertian model, where the cylinder represents the ith LED, and the rectangular prism denotes the $P D$.

where $m_{i}$ is the Lambertian order for the ith LED transmitter, $A_{\mathrm{R}}$ is the area of the PD at the VLC receiver, and $\phi_{i}$ and $\theta_{i}$ are the irradiation and the incidence angles, respectively, between the ith LED transmitter and the VLC receiver [46], [47]. From the definitions of $\phi_{i}$ and $\theta_{i}$ (see Fig. 2), (3) can be rewritten as

$$
\alpha_{i}=-\frac{\left(m_{i}+1\right) A_{\mathrm{R}}\left[\left(\boldsymbol{l}_{\mathrm{r}}-\boldsymbol{l}_{\mathrm{t}}^{i}\right)^{T} \boldsymbol{n}_{\mathrm{t}}^{i}\right]^{m_{i}}\left(\boldsymbol{l}_{\mathrm{r}}-\boldsymbol{l}_{\mathrm{t}}^{i}\right)^{T} \boldsymbol{n}_{\mathrm{r}}}{2 \pi\left\|\boldsymbol{l}_{\mathrm{r}}-\boldsymbol{l}_{\mathrm{t}}^{i}\right\|^{m_{i}+3}}
$$

where $\boldsymbol{n}_{\mathrm{r}}=\left[\begin{array}{lll}n_{\mathrm{r}, 1} & n_{\mathrm{r}, 2} & n_{\mathrm{r}, 3}\end{array}\right]^{T}$ and $\boldsymbol{n}_{\mathrm{t}}^{i}=\left[\begin{array}{lll}n_{\mathrm{t}, 1}^{i} & n_{\mathrm{t}, 2}^{i} & n_{\mathrm{t}, 3}^{i}\end{array}\right]^{T}$ stand for the orientation vectors of the VLC receiver and the ith LED transmitter, respectively [47], [55].

It is assumed that the parameters $A_{\mathrm{R}}, R_{p}, \boldsymbol{n}_{\mathrm{r}}, m_{i}, \boldsymbol{l}_{\mathrm{t}}^{\mathrm{i}}$, and $\boldsymbol{n}_{\mathrm{t}}^{i}$, and the transmitted signals $s_{i}(t)$ for $i=1, \ldots, N_{\mathrm{L}}$ are known by the VLC receiver [55], [63].

Remark: It is important to emphasize that the signal model in VLP systems differs from the one in RF-based localization systems since the intensity of the electromagnetic waves is modulated in VLP systems instead of the field of the wave, which is employed in RF-based localization systems [47]. Therefore, unlike in RF-based systems, the transmitted signal in VLP systems cannot be negative. Hence, the design of modulation techniques to be employed in VLP systems necessitates the consideration of optical signal properties together with illumination constraints. In addition to modulation techniques, VLP- and RF-based systems differ in the channel model, as well. In VLP systems, the optical channel model is considered, which significantly depends on the orientations of the LED transmitter and the VLC receiver, and the area of the PD at the VLC receiver besides the locations of the VLC receiver and the LED transmitter.

2) Direct-Positioning-Based ML Estimators: The rationale behind the use of direct positioning is to estimate the VLC receiver location $\boldsymbol{l}_{\mathrm{r}}$ by exploiting all the available information about $\boldsymbol{l}_{\mathrm{r}}$, i.e., the received signals $\left\{r_{i}(t)\right\}_{i=1}^{N_{\mathrm{L}}}$ in (1). In this way, the information loss stemming from intermediate steps (cf., 
Section II-B) can be avoided and the location can be estimated in an optimal manner. In the following, the direct-positioning-based ML estimators are investigated for synchronous, quasi-synchronous, and asynchronous VLP systems.

- Direct positioning in synchronous systems: Since $\Delta_{i}=0$ in (2) for synchronous systems, the ML estimator for $\boldsymbol{l}_{\mathrm{r}}$ can be obtained from (1) as [63]

$$
\begin{aligned}
& \hat{\boldsymbol{l}}_{\mathrm{r}}^{\mathrm{DP} \text {,syn }}=\underset{\boldsymbol{l}_{\mathrm{r}}}{\arg \max _{i=1}} \sum_{i=1}^{N_{\mathrm{L}}} \alpha_{i} \int_{T_{1, i}}^{T_{2, i}} r_{i}(t) s_{i}\left(t-\tau_{i}\right) d t \\
& -\frac{R_{p}}{2} \sum_{i=1}^{N_{L}} \alpha_{i}^{2} E_{2}^{i}
\end{aligned}
$$

where $E_{2}^{i}$ is the electrical energy of $s_{i}(t)$, defined as

$$
E_{2}^{i} \triangleq \int_{0}^{T_{s, i}}\left(s_{i}(t)\right)^{2} d t .
$$

The direct estimator in (5) performs a search over all possible values of the unknown location $\boldsymbol{l}_{\mathrm{r}}$ via its relation to $\tau_{i}$ in (2) (with $\Delta_{i}=0$ ) and to $\alpha_{i}$ in (4).

- Direct positioning in quasi-synchronous systems: As the LED transmitters are synchronized to a common time base in quasi-synchronous systems, the time offsets in (2) are the same among the LED transmitters, i.e., $\Delta_{i}=\Delta$ for $i=1, \ldots, N_{\mathrm{L}}$, where $\Delta$ is an unknown time offset. Under this setting, the direct-positioningbased ML estimator is given by [94]

$$
\begin{aligned}
\left(\hat{\boldsymbol{l}}_{\mathrm{r}}^{\mathrm{DP}, \mathrm{qsy}}, \hat{\Delta}\right)= & \underset{\left(\boldsymbol{l}_{\mathrm{r}}, \Delta\right)}{\arg \max } \sum_{i=1}^{N_{\mathrm{L}}} \alpha_{i} \int_{T_{1, i}}^{T_{2, i}} r_{i}(t) s_{i}\left(t-\tau_{\mathrm{i}}\right) d t \\
& -\frac{R_{p}}{2} \sum_{i=1}^{N_{\mathrm{L}}} \alpha_{i}^{2} E_{2}^{i}
\end{aligned}
$$

where $E_{2}^{i}$ is as defined in (6). Since the time offset $\Delta$ between the receiver and the transmitters is unknown, a joint search over $\boldsymbol{l}_{\mathrm{r}}$ and $\Delta$ must be performed in (7) to find the optimal $\boldsymbol{l}_{\mathrm{r}}$. Note that $\alpha_{i}$ in (7) depends on $\boldsymbol{l}_{\mathrm{r}}$ via (4), while $\tau_{i}$ is a function of both $\boldsymbol{l}_{\mathrm{r}}$ and $\Delta$ via (2) (with $\Delta_{i}=\Delta$ for $i=1$, $\left.\ldots, N_{\mathrm{L}}\right)$.

- Direct positioning in asynchronous systems: For asynchronous systems, the time offset $\Delta_{i}$ in (2) is an unknown parameter. In this case, the ML estimator is obtained as [63]

$$
\hat{\boldsymbol{l}}_{\mathrm{r}}^{\mathrm{DP}, \text { asy }}=\arg \max _{\boldsymbol{l}_{\mathrm{r}}} \sum_{i=1}^{N_{\mathrm{L}}}\left(\alpha_{i} \tilde{C}_{r s}^{i}-0.5 R_{p} \alpha_{i}^{2} E_{2}^{i}\right)
$$

where $E_{2}^{i}$ is given by (6) and

$$
\tilde{C}_{r s}^{i} \triangleq \max _{\tau_{i}} \int_{T_{1, i}}^{T_{2, i}} r_{i}(t) s_{i}\left(t-\tau_{i}\right) d t .
$$

As observed from (8), the direct estimator in asynchronous systems attempts to determine the location of the VLC receiver based on its relation with the channel attenuation factor in (4). This is due to the fact that no information about $\boldsymbol{l}_{\mathrm{r}}$ can be extracted from $\tau_{i}$ 's in (2) due to the unknown time offsets in the asynchronous case. For this reason, the resulting estimator in (8) employs the correlator peak in (9) [cf., the integral expression in (5)] and utilizes the relation of $\boldsymbol{l}_{\mathrm{r}}$ to $\alpha_{i}^{\prime}$ 's only.

The direct positioning estimators in (5), (7), and (8) constitute the optimal estimators (in the ML sense) for the location of the VLC receiver. However, the direct positioning paradigm may have several drawbacks, including high computational burden [63] and excessive data storage and communication concerns.

Remark: In addition to their utilization in VLP systems, direct localization algorithms have also been widely used for RF-based localization systems in the literature [82]-[88]. A common observation in RF and VLP systems regarding the performance of direct position estimation is that the improvement in localization accuracy provided by direct positioning over its two-step counterpart ${ }^{2}$ is particularly significant in the low signal-to-noise ratio (SNR) regime [63], [82], [83], [85], [86], [88], [94]. The major difference between these two systems lies in the fact that different types of signal metrics are employed for position estimation. In particular, both signal-strength-based (i.e., $\alpha_{i}$ ) and time-based (i.e., $\tau_{i}$ and $\Delta$ ) information are utilized for direct positioning in synchronous and quasisynchronous VLP systems, as seen from (5) and (7). On the other hand, received powers of RF signals can severely be affected by multipath and shadowing effects [95]; hence, they cannot be used reliably in RF-based direct positioning algorithms. Hence, in general, direct localization approaches in RF systems consider either synchronous [82]-[85] or quasi-synchronous [83], [87] scenarios. However, for VLP systems, direct position estimation can be performed also for asynchronous scenarios since the Lambertian model in (3) can accurately characterize the optical channel attenuation [8].

3) Performance Limits: Theoretical performance limits provide essential guidelines for the design and evaluation of practical VLP systems. In this part, we present the CramérRao lower bound (CRLB) on variances of unbiased estimates of $\boldsymbol{l}_{\mathrm{r}}$ in synchronous, quasi-synchronous, and asynchronous VLP systems. The localization accuracy limits characterized by the presented CRLBs are attainable by the corresponding ML estimators in (5), (7), and (8) at high SNRs and/or bandwidths [96], [97].

- CRLB in synchronous systems: The CRLB for localization of the VLC receiver in synchronous VLP systems is expressed as [63]

$$
\mathbb{E}\left\{\left\|\hat{\boldsymbol{l}}_{\mathrm{r}}-\boldsymbol{l}_{\mathrm{r}}\right\|^{2}\right\} \geq \operatorname{trace}\left\{\mathrm{J}_{\text {syn }}^{-1}\right\}
$$

${ }^{2}$ See Section II-B for detailed treatment on two-step positioning techniques. 
where $\hat{\boldsymbol{l}}_{\mathrm{r}}$ represents an unbiased estimate for the location $\boldsymbol{l}_{\mathrm{r}}$ and the Fisher information matrix (FIM) $\mathbf{J}_{\text {syn }}$ is calculated from

$$
\begin{aligned}
{\left[\mathrm{J}_{\mathrm{syn}}\right]_{k_{1}, k_{2}}=\frac{R_{p}^{2}}{\sigma^{2}} \sum_{i=1}^{N_{\mathrm{L}}}(} & E_{2}^{i} \frac{\partial \alpha_{i}}{\partial l_{\mathrm{r}, k_{1}}} \frac{\partial \alpha_{i}}{\partial l_{\mathrm{r}, k_{2}}}+E_{1}^{i} \alpha_{i}^{2} \frac{\partial \tau_{i}}{\partial l_{\mathrm{r}, k_{1}}} \frac{\partial \tau_{i}}{\partial l_{\mathrm{r}, k_{2}}} \\
& \left.-E_{3}^{i} \alpha_{i}\left(\frac{\partial \alpha_{i} \partial \tau_{i}}{\partial l_{\mathrm{r}, k_{1}} \partial l_{\mathrm{r}, k_{2}}}+\frac{\partial \tau_{i}}{\partial l_{\mathrm{r}, k_{1}} \partial \alpha_{\mathrm{r}, k_{2}}}\right)\right)
\end{aligned}
$$

for $k_{1}, k_{2} \in\{1,2,3\}$. In (11), $\partial \alpha_{i} / \partial l_{\mathrm{r}, k}$ and $\partial \tau_{i} / \partial l_{\mathrm{r}, k}$ are, respectively, the partial derivatives of the channel attenuation in (4) and the TOA parameter in (2) (for $\left.\Delta_{i}=0\right)$ with respect to $l_{\mathrm{r}, k}, E_{2}^{i}$ is given by (6), and $E_{1}^{i}$ and $E_{3}^{i}$ are defined as

$$
\begin{gathered}
E_{1}^{i} \triangleq \int_{0}^{T_{s, i}}\left(s^{\prime}{ }_{i}(t)\right)^{2} d t \\
E_{3}^{i} \triangleq \int_{0}^{T_{s, i}} s_{i}(t) s_{i}^{\prime}(t) d t
\end{gathered}
$$

with $s_{i}^{\prime}(t)$ denoting the derivative of $s_{i}(t)$.

- CRLB in quasi-synchronous systems: In quasi-synchronous VLP systems, the CRLB for location estimation of the VLC receiver is expressed as [94]

$$
\mathbb{E}\left\{\left\|\hat{\boldsymbol{l}}_{\mathrm{r}}-\boldsymbol{l}_{\mathrm{r}}\right\|^{2}\right\} \geq \operatorname{trace}\left\{\mathbf{J}_{\mathrm{qsy}}^{-1}\right\}
$$

where

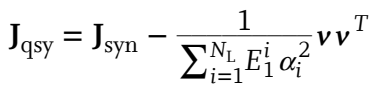

with $\mathbf{J}_{\text {syn }}$ being given by (11), $v=\left[\begin{array}{lll}v_{1} & v_{2} & v_{3}\end{array}\right]^{T}$, and

$$
v_{k} \triangleq \frac{R_{p}}{\sigma} \sum_{i=1}^{N_{\mathrm{L}}}\left(E_{1}^{i} \alpha_{i}^{2} \frac{\partial \tau_{i}}{\partial l_{\mathrm{r}, k}}-E_{3}^{i} \alpha_{i} \frac{\partial \alpha_{i}}{\partial l_{\mathrm{r}, k}}\right)
$$

for $k \in\{1,2,3\}$.

- CRLB in asynchronous systems: For asynchronous VLP systems, the CRLB is stated as [55], [63]

$$
\mathbb{E}\left\{\left\|\hat{\boldsymbol{l}}_{\mathrm{r}}-\boldsymbol{l}_{\mathrm{r}}\right\|^{2}\right\} \geq \operatorname{trace}\left\{\mathrm{J}_{\text {asy }}^{-1}\right\}
$$

where the FIM is calculated from

$$
\begin{aligned}
& \left.\qquad \mathrm{J}_{\mathrm{asy}}\right]_{k_{1}, k_{2}}=\frac{R_{p}^{2}}{\sigma^{2}} \sum_{i=1}^{N_{\mathrm{L}}}\left(E_{2}^{i}-\frac{\left(E_{3}^{i}\right)^{2}}{E_{1}^{i}}\right) \frac{\partial \alpha_{i}}{\partial l_{\mathrm{r}, k_{1}}} \frac{\partial \alpha_{i}}{\partial l_{\mathrm{r}, k_{2}}} \\
& \text { for } k_{1}, k_{2} \in\{1,2,3\} .
\end{aligned}
$$

It is noted that the transmitted signals $s_{i}(t)$ affect the FIM expressions in (11), (15), and (18) via $E_{1}^{i}, E_{2}^{i}$, and $E_{3}^{i}$, and the contribution of the system geometry to the FIM is through the $\partial \alpha_{i} / \partial l_{r, k}$ and $\partial \tau_{i} / \partial l_{r, k}$ terms. In addition, the $\partial \tau_{i} / \partial l_{r, k}$ terms do not appear in (18) since the TOA parameter does not provide location related information in asynchronous systems.

Based on the CRLB expressions, some practical scenarios can be investigated to gain insights into the localization performance of VLP systems with varying levels of synchronism. In particular, the following practical assumptions are considered: 1) $E_{3}^{i}=0$ for $i=1, \ldots, N_{\mathrm{L}}$, which is the case for most practical pulses, ${ }^{3}$ and 2$)$ the transmitted signals $s_{i}(t)$ are identical, i.e., $s_{i}(t)=s(t)$. (In this case, the parameters related to the pulse shape are the same for all the LED transmitters, i.e., $E_{1}^{i}=E_{1}, E_{2}^{i}=E_{2}$, and $E_{3}^{i}=E_{3}$ for $i=1, \ldots, N_{\mathrm{L}}$.) Then, it follows from (15) that

$$
\mathbf{J}_{\text {syn }}-\mathbf{J}_{\mathrm{qsy}}=\frac{R_{p}^{2} E_{1}}{\sigma^{2}} \vartheta \vartheta^{T}
$$

where $\vartheta=\left[\vartheta_{1} \vartheta_{2} \vartheta_{3}\right]^{T}$ with $\vartheta_{k} \triangleq \sum_{i=1}^{N_{\mathrm{L}}} \alpha_{i}^{2}\left(\partial \tau_{i} / \partial l_{\mathrm{r}, k}\right) / \sqrt{\sum_{i=1}^{N_{\mathrm{L}}} \alpha_{i}^{2}}$ for $k \in\{1,2,3\}$. From (19), it is clear that $\mathbf{J}_{\text {syn }} \geqslant \mathbf{J}_{\text {qsy }}$ is always satisfied since $E_{1}$ is positive by definition, where $\mathbf{J}_{\text {syn }} \geqslant \mathbf{J}_{\text {qsy }}$ means that $\mathbf{J}_{\text {syn }}-\mathbf{J}_{\mathrm{qsy}}$ is positive semidefinite. Therefore, it is deduced from (10) and (14) that synchronism between the LED transmitters and the VLC receiver helps achieve a reduced CRLB (that is, improved localization performance), as expected. In addition, based on Parseval's relation [48]

$$
E_{1}=4 \pi^{2} \beta^{2} E_{2}
$$

where $\beta$ denotes the effective bandwidth of $s(t),{ }^{4}$ it can be inferred from (19) that the information gain via synchronism becomes more significant as $\beta$ gets larger. Similar to (19), a relation between the FIMs of quasi-synchronous and asynchronous systems can be derived as

$$
\mathrm{J}_{\mathrm{qsy}}-\mathrm{J}_{\mathrm{asy}}=\frac{R_{p}^{2} E_{1}}{\sigma^{2}}\left(\left(\sum_{i=1}^{N_{\mathrm{L}}} \alpha_{i}^{2} \boldsymbol{\mu}_{i} \boldsymbol{\mu}_{i}^{T}\right)-\frac{1}{\sum_{i=1}^{N_{\mathrm{L}} \alpha_{i}^{2}}} \widetilde{\boldsymbol{\mu}} \widetilde{\mu}^{T}\right)
$$

where $\mu_{i}=\left[\mu_{i, 1} \mu_{i, 2} \mu_{i, 3}\right]^{T}, \tilde{\mu} \triangleq \sum_{i=1}^{N_{\mathrm{L}}} \alpha_{i}^{2} \mu_{i}$, and $\mu_{i, k} \triangleq \partial \tau_{i} / \partial l_{\mathrm{r}, k}$. It follows from the Cauchy-Schwarz inequality that $\mathbf{J}_{\mathrm{qsy}} \geqslant \mathbf{J}_{\mathrm{asy}}$. Based on (21), similar conclusions to those related to (19) can be made. Namely, as the effective bandwidth increases, the accuracy of (quasi-)synchronous positioning improves. This suggests that using LEDs with optical clock rates up to $120 \mathrm{MHz}$ [98], very precise position estimates can be obtained in (quasi-)synchronous VLP systems. However, for VLP signals with relatively low effective bandwidths and/ or in the presence of imperfect synchronization, asynchronous VLP systems would be more preferable due to their low complexity.

\section{B. Two-Step Positioning}

As the most prevalent approach for positioning in VLP systems, the two-step method first extracts position related parameters from the received VLC signals and then performs position estimation based on those parameters. Compared to direct positioning, the two-step approach leads to low computational complexity as it utilizes only a subset of the available information (i.e., position-dependent parameters) for positioning instead of the entire received signals [63].

${ }^{3} E_{3}^{i}$ is calculated from (13) as $E_{3}^{i}=\left(s_{i}\left(T_{s, i}\right)^{2}-s_{i}(0)^{2}\right) / 2$, which is zero for practical pulse shapes (e.g., [47, eq. (3)]).

${ }^{4} \beta$ is defined as $\beta=\sqrt{\left(1 / E_{2}\right) \int f^{2}|S(f)|^{2} d f}$ with $S(f)$ representing the Fourier transform of $s(t)[62]$. 
Hence, a certain level of accuracy is sacrificed for the sake of a reduced computational burden in two-step positioning. In the following, we first describe the commonly employed position-dependent parameters in the first step and how to estimate them in an optimal manner. Then, we present position estimation techniques that employ those parameters in the second step to obtain an estimate of the VLC receiver location.

1) Parameter Estimation: Estimation of positiondependent parameters in a VLP system is carried out as the first step of a two-step positioning method. This part will focus on the most common parameters used in the first step of VLP algorithms, including RSS, TOA, TDOA, and AOA, and also present hybrid algorithms that utilize a combination of those parameters.

a) Received Signal Strength: The RSS estimate (measurement) obtained from the received VLC signal contains positional information as the channel attenuation factor $^{5}$ in (4) depends on the location $\boldsymbol{l}_{\mathrm{r}}$ of the VLC receiver. According to the Lambertian model in (4), which characterizes the level of attenuation in visible light channels, the received signal gets weaker as the distance between the LED transmitter and the VLC receiver increases or as the displacement vector $\boldsymbol{l}_{\mathrm{r}}-\boldsymbol{l}_{\mathrm{t}}^{i}$ deviates from the orientation of the LED transmitter and/or the VLC receiver. In practical VLP systems, RSS-based positioning is a common technique due to its low-cost hardware implementation that requires no synchronization, as opposed to TOA-based schemes [44]-[46]. In addition, multipath effects in indoor visible light channels are not significant as compared to RF propagation, which makes the Lambertian formula a reliable model for quantification of channel attenuation [16]-[18], [8, Sec. 3.4.1]. ${ }^{6}$ Therefore, RSS has been a popular discriminative feature for positioning in visible light systems [40], [44]-[46], [64], [100]-[103].

For an asynchronous VLP system, the ML estimate $\hat{\alpha}_{i}$ of the RSS parameter $\alpha_{i}$ corresponding to the ith LED transmitter can be obtained from the received signal in (1) as

$$
\hat{\alpha}_{i}=\frac{\tilde{C}_{r s}^{i}}{R_{p} E_{2}^{i}}
$$

if $\tilde{C}_{r s}^{i} \geq 0\left(\hat{\alpha}_{i}=0\right.$ otherwise $)$, where $\tilde{C}_{r s}^{i}$ and $E_{2}^{i}$ are given by (9) and (6), respectively [63]. From (22), it is observed that the RSS parameter corresponds to a scaled version of the measured energy at the VLC receiver as $\tilde{C}_{r s}^{i}$ represents the peak value of the correlator output in (9) (obtained by correlating the received signal with delayed replicas of the transmitted signal). After obtaining the RSS

\footnotetext{
${ }^{5}$ In this paper, the RSS parameter refers to the channel attenuation factor $\alpha_{i}$ since $\alpha_{i}$ is nonnegative and the received signal energy is determined by $\alpha_{i}$ [63].

${ }^{6}$ Multipath effects can significantly be mitigated by employing calibration techniques, such as selecting a subset of LEDs and using a dense LED configuration [99].
}

estimates $\left\{\hat{\alpha}_{i}\right\}_{i=1}^{N_{\mathrm{L}}}$ in the first step, a two-step algorithm can use them as an input to the second step to estimate the VLC receiver location.

Since the orientations of the LED transmitters and the VLC receiver (denoted by $\boldsymbol{n}_{\mathrm{t}}^{i}$ and $\boldsymbol{n}_{\mathrm{r}}$, respectively) affect the RSS measurement in (4), the estimated RSS value cannot directly be translated to a distance estimate in general. However, in certain practical scenarios, distance information can unambiguously be obtained from the RSS estimates. For example, consider a VLP scenario in which the LED transmitters are pointing downwards (i.e., $\boldsymbol{n}_{\mathrm{t}}^{i}=\left[\begin{array}{lll}0 & 0 & -1\end{array}\right]^{\mathrm{T}}$ ), the VLC receiver is pointing upwards (i.e., $\boldsymbol{n}_{\mathrm{r}}=\left[\begin{array}{lll}0 & 0 & 1\end{array}\right]^{T}$ ), and the height of the VLC receiver is known (that is, the receiver moves on a horizontal plane and performs 2-D localization) [45], [47]-[49], [64], [100], [101]. Then, the RSS parameter in (4) can be expressed as

$$
\alpha_{i}=\frac{\left(m_{i}+1\right) A_{\mathrm{R}} h_{i}^{m_{i}+1}}{2 \pi d_{i}^{m_{i}+3}}
$$

where $h_{i}$ is the height of the ith LED transmitter with respect to the VLC receiver and $d_{i}=\left\|\boldsymbol{l}_{\mathrm{r}}-\boldsymbol{l}_{\mathrm{t}}^{i}\right\|$ is the distance between the ith LED transmitter and the VLC receiver. Based on the relation (23), the distance estimate $\hat{d}_{i}$ can be calculated from the RSS estimate $\hat{\alpha}_{i}$ [40], [48], which can then be utilized in a trilateration algorithm to get the final position estimate [18], [44], [45], [104].

The accuracy of RSS information can be quantified by theoretical performance limits to explore the best achievable estimation performance. The CRLB on the variance of an unbiased estimate $\hat{\alpha}_{i}$ of the RSS parameter $\alpha_{i}$ can be expressed as [63]

$$
\mathbb{E}\left\{\left(\hat{\alpha}_{i}-\alpha_{i}\right)^{2}\right\} \geq \frac{E_{1}^{i}}{E_{1}^{i} E_{2}^{i}-\left(E_{3}^{i}\right)^{2}} \frac{\sigma^{2}}{R_{p}^{2}}
$$

where $E_{1}^{i}, E_{2}^{i}$, and $E_{3}^{i}$ are given by (12), (6), and (13), respectively. As noted from (24), the performance of RSS estimation deteriorates with an increase in the noise level in the received signal in (1). In addition, as the electrical energy $E_{2}^{i}$ of the transmitted signal increases, the RSS information becomes more accurate due to more favorable SNR conditions. Moreover, the accuracy of RSS estimates improves for larger values of the responsivity $R_{p}$ of the $\mathrm{PD}$, since responsivity, defined in terms of amperes per watt, measures the conversion efficiency of incident optical power to electrical current [93].

For scenarios in which the RSS-distance transformation can explicitly be performed [as in (23)], the CRLB for RSSbased distance estimation can be obtained as [48], [101]

$$
\begin{aligned}
\mathbb{E}\left\{\left(\hat{d}_{i}-d_{i}\right)^{2}\right\} & \frac{E_{1}^{i}}{E_{1}^{i} E_{2}^{i}-\left(E_{3}^{i}\right)^{2}}\left(\frac{2 \pi \sigma d_{i}^{3}}{R_{p} A_{\mathrm{R}}}\right)^{2} \\
& \times \frac{1}{\left(m_{i}+1\right)^{2}\left(m_{i}+3\right)^{2}}\left(\frac{d_{i}}{h_{i}}\right)^{2 m_{i}+2}
\end{aligned}
$$


where $\hat{d}_{i}$ denotes an unbiased estimate of $d_{i}$. From (25), it is observed that when $d_{i} \approx h_{i}$, that is, when the VLC receiver is almost directly under the LED, the CRLB decreases with an increasing Lambertian order $m_{i}$ since the sensitivity of RSS to distance becomes more pronounced for larger values of $m_{i}$ in such cases. More specifically, considering the fact that the directivity of an LED is determined by the Lambertian order (more directive for larger orders), the Lambertian pattern in (23) is more sensitive to distance (or, angle ${ }^{7}$ ) around the peak point of the pattern for higher levels of directivity. On the other hand, when $d_{i} \gg h_{i}$, the accuracy of distance estimation improves as the Lambertian order decreases since more signal power can be received at longer distances if the LED is not very directive. These remarks are illustrated for an example scenario in Fig. 3, where $h_{i}=2 \mathrm{~m}, E_{2}^{i}=2.25 \times$ $10^{-4} \mathrm{~W}, E_{3}^{i}=0, \sigma^{2}=1.3381 \times 10^{-22} \mathrm{~W} / \mathrm{Hz}, R_{p}=0.4 \mathrm{~A} / \mathrm{W}$, and $A_{\mathrm{R}}=10^{-4} \mathrm{~m}^{2}$ in (25) (the same parameters as in [48] with a source optical power of $1 \mathrm{~W})$.

b) Time of Arrival: The distance between an LED transmitter and a VLC receiver can be calculated based on the time of flight of the signal between the two devices, which is determined by the TOA parameter as defined in (2). The primary requirement for the utilization of TOA information in VLP systems is that the clocks of the LED transmitters and the VLC receiver must be synchronized [47]. In the case of synchronization, the VLC receiver can estimate the TOAs of the incoming signals from multiple LEDs and accomplish position estimation based on these TOA (equivalently, distance) estimates. Due to the increased cost of implementation associated with clock synchronization, the research on TOA-based positioning is fairly limited [47]-[49], [63].

${ }^{7}$ For 2-D scenarios, irradiation angle $\varphi_{i}$ can be converted to distance by $d_{i}=h_{i} / \cos \varphi_{i}$, where $h_{i}$ is the known height of the LED with respect to the receiver.

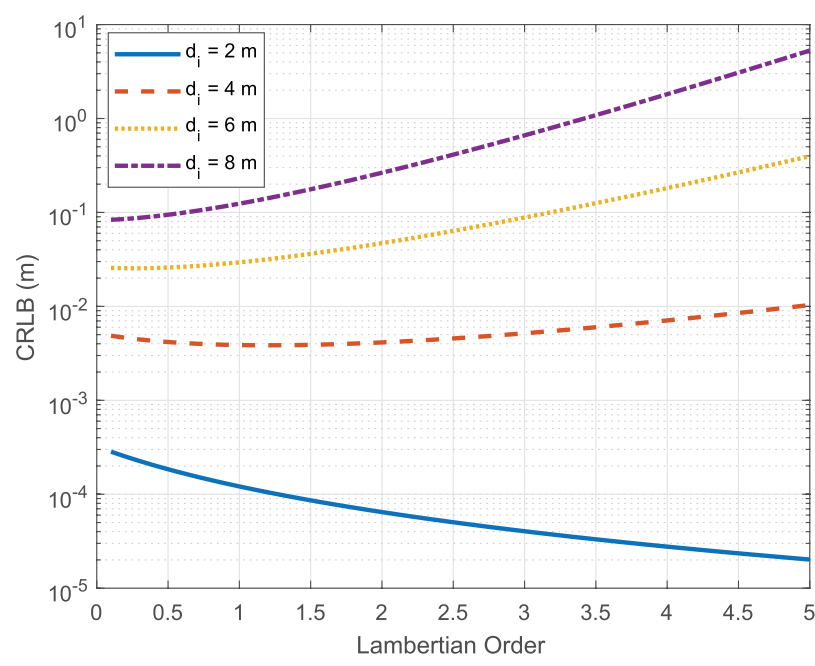

Fig. 3. The square root of the CRLB expression in (25) versus the Lambertian order $m_{i}$, for various values of distance $d_{i}$.
The TOA parameter can be estimated from the received VLC signal in (1) as [48], [63]

$$
\hat{\tau}_{i}=\arg \max _{\tau_{i}} \int_{T_{1, i}}^{T_{2, i}} r_{i}(t) s_{i}\left(t-\tau_{i}\right) d t
$$

where $\hat{\tau}_{i}$ is the ML estimate of the TOA parameter $\tau_{i}$. As observed from (26), the optimal ML TOA estimation is achieved by the correlation (matched filter) receiver [105]. That is, the TOA between the transmitter and the receiver is estimated by performing correlation of the received VLC signal with delayed copies of the transmit signal and identifying the location at which the peak occurs.

The CRLB for distance estimation based on TOA information in synchronous VLP systems is stated as [47], [48]

$$
\mathbb{E}\left\{\left(\hat{d}_{i}-d_{i}\right)^{2}\right\} \geq \frac{E_{2}^{i}}{E_{1}^{i} E_{2}^{i}-\left(E_{3}^{i}\right)^{2}}\left(\frac{\sigma c}{R_{p} \alpha_{i}}\right)^{2}
$$

where $\hat{d}_{i}$ represents an unbiased estimate of distance $d_{i}$ between the ith LED transmitter and the VLC receiver, and $c$ is the speed of light. It is noted from (27) that the accuracy of TOA estimation increases with the SNR [cf., the signal model in (1)]. In addition, for $E_{3}^{i}=0$, the lower bound in (27) reduces for larger effective bandwidths via (20). As opposed to the TOA-based method, the effective bandwidths $\beta_{i}$ 's have no effects on the CRLB of RSS-based distance estimation in (25) for $E_{3}^{i}=0$. Therefore, synchronous VLP systems exploiting high-bandwidth LEDs offer the potential of high accuracy distance estimation.

c) Time Difference of Arrival: TDOA-based positioning exploits the differences between the distances from multiple LED transmitters to the VLC receiver. The TDOA parameter can be obtained by taking the difference of two TOA measurements corresponding to two different LEDs as

$$
\Delta \hat{\tau}_{i j}=\hat{\tau}_{i}-\hat{\tau}_{j}
$$

where $\Delta \hat{\tau}_{i j}$ denotes the TDOA estimate between the $i$ th and $j$ th LEDs, and $\hat{\tau}_{i}$ is the TOA estimate for the $i$ th LED as in (26). Considering the TOA model in (2), the TDOA parameter in the noiseless case can be modeled as follows:

$$
\Delta \tau_{i j}=\frac{\left\|\boldsymbol{l}_{\mathrm{r}}-\boldsymbol{l}_{\mathrm{t}}^{\mathrm{i}}\right\|}{c}-\frac{\left\|\boldsymbol{l}_{\mathrm{r}}-\boldsymbol{l}_{\mathrm{t}}^{\mathrm{t}}\right\|}{c}+\Delta_{i}-\Delta_{j} .
$$

In the presence of synchronization among the LED transmitters (i.e., $\Delta_{i}=\Delta$ for $i \in\left\{1, \ldots, N_{\mathrm{L}}\right\}$ ), the TDOA information in (29) characterizes the difference in the TOA values belonging to the two LEDs since the constant time offsets are removed via subtraction. Hence, utilizing TDOA measurements requires that the LED transmitters be synchronized to a common time reference [106]. However, as opposed to TOA-based positioning, no synchronization is required between the LED transmitters and the VLC 
receiver, thereby making TDOA a relatively less expensive method for VLP systems [106].

Besides the TDOA estimation method in (28), the cross correlation of the two received signals can be performed to estimate the TDOA parameter as follows [50], [51]:

$$
\Delta \hat{\tau}_{i j}=\arg \max _{\Delta \tau_{i j}} \int_{T_{1, i j}}^{T_{2, i j}} r_{i}(t) r_{j}\left(t+\Delta \tau_{i j}\right) d t
$$

where $T_{1, i j}$ and $T_{2, i j}$ specify the complete observation interval corresponding to the signals emitted by the ith and $j$ th LEDs. Another method for measuring the range differences between LEDs is to utilize different carrier frequencies for the transmit signals $s_{i}(t)$ belonging to different LEDs [26], [52], [107]. In this way, phase differences between the received signals can be converted to distance differences [52]. Moreover, TDOA can also be calculated by using the same carrier frequency with different phase shifts for $s_{i}(t)$ 's [108].

d) Angle of Arrival: The information regarding the AOA of VLC signals incident upon the PD of the VLC receiver can be exploited to determine the position of the VLC receiver. Specifically, with the knowledge of the positions and the orientations of LED transmitters, the AOA (i.e., the incidence angle) of a signal arriving at a PD yields a unique angle of departure (AOD) (i.e., the irradiation angle) from the LED that emits that signal. In the ideal case of noiseless AOA/AOD estimation, the intersection point of the lines extending from multiple LEDs in the directions of the corresponding AOD estimates would coincide with the location of the VLC receiver [55], [60], [109]. Hence, VLP systems can take advantage of the AOA parameter for location estimation.

One way to estimate the AOA parameter is via the LED connectivity information, which can be acquired by deploying multi-LED visible light access points (VAPs) in an indoor scenario [55], [60]. Each VAP can be designed to contain multiple LED transmitters with very narrow field of views, whose orientations are adjusted such that every point in the room gathers a signal only from a single LED [60, Fig. 1]. For such a configuration, the receiver is connected to a single LED of each VAP, which characterizes the AOA information gathered from that VAP via the orientation of the designated LED.

Another method for AOA estimation is to use an array of PDs in the VLC receiver [53], [54], [110], [111]. For instance, the differences in RSS measurements at the PDs arranged in a circular layout on the VLC receiver can be utilized to estimate the AOA of the signal at the receiver [53]. It is also possible to use a uniform linear array (ULA) of PDs to determine the direction of arrival of the VLC signal via a beamforming vector [54], [110]. To increase the sensitivity of received powers at PDs to the direction of signal arrival, a reasonable approach is to employ aperture-based receivers where an opaque aperture containing a hole is placed on top of each PD in a circular array [56], [111]. In such configurations, the holes on the apertures are shifted from the location of PDs toward the center of the circular array [111, Fig. 1] to enlarge the relative differences in the measured signal powers at PDs, resulting in more accurate AOA estimates.

Similar to array configurations, the VLC receiver structures involving multiple PDs with different orientations can be employed to obtain AOA information from the received signals [109], [112]-[115]. Using a corner-cube structure that involves three PDs with orthogonal detector planes, AOA estimation can be performed by measuring the difference in signal powers received at the PDs [109], [115]. Similarly, multiple tilted PDs on a VLC receiver can reveal information about the direction of arrival of incoming signals via the differences of RSS measurements at the PDs [112]-[114].

e) Hybrid Approaches: In addition to parameter estimation methods that utilize only a single property of received VLC signals (e.g., TOA, RSS, or AOA), there exist hybrid schemes that aim to estimate position-dependent parameters through joint utilization of several signal attributes [48]. Such hybrid approaches are likely to produce more accurate first-step parameter estimates compared to conventional techniques by blending the benefits of each signal characteristic into a unified estimation framework.

As discussed previously, both TOA and RSS measurements can provide information for distance estimation in VLP systems. Hence, an ML-based distance estimator that incorporates information from both time delay (i.e., TOA) and optical channel attenuation (i.e., RSS) parameters can be designed to improve the accuracy of ranging in a synchronous scenario [48]. For such an estimator, the information obtained from the TOA parameter gets more significant as the effective bandwidth of the signal increases. For small effective bandwidths (around for 1 $\mathrm{MHz}$ or lower), the additional information from the TOA parameter becomes negligible compared to the information obtained from the RSS parameter; hence, synchronism does not provide significant performance benefits in such scenarios (since RSS can also be estimated in asynchronous systems) [48].

2) Position Estimation: As the second step of a two-step positioning algorithm, the position estimation procedure takes as input the position related parameters from the first step and outputs the estimated position of the VLC receiver. In this part, we discuss four different classes of position estimation methods, namely, proximity-based methods, geometric methods, statistical methods, and fingerprinting/ mapping methods.

a) Proximity-Based Methods: Proximity-based position estimation depends simply on connectivity information and 
thus has the advantage of being computationally efficient. In a proximity-based positioning scheme, the VLC receiver extracts from the received signals the identification data of the nearest $\operatorname{LED}^{8}$ and relays it to a central database, which transmits the LED position information corresponding to that identification back to the receiver [1], [57], [58], [116]. As an alternative approach, LED transmitters may broadcast their positions instead of their identities, which circumvents the need for communications with the database [1], [58]. As the result of this process, the VLC receiver acquires the location of the closest LED, which provides a rough location information (i.e., the receiver lies in the area spanned by the field of view of the specified LED transmitter). Therefore, the accuracy of proximity based positioning is primarily affected by how dense the LEDs are deployed over the ceiling of the indoor environment in which the receiver operates [64].

Proximity-based localization is particularly useful for applications where accuracy requirements are not strict, such as asset tracking in hospitals and location-aware services in art galleries [1]. However, certain applications, such as mobile robot navigation, may require more precise location information than that provided by the proximity-based method [1]. In order to alleviate this problem, angular information obtained from auxiliary sensors can be combined with the proximity-based information to refine the coarse position estimates [117]. In addition, a biconvex lens can be inserted under each LED luminary containing multiple LED transmitters to improve the accuracy of proximity-based positioning by providing angular diversity [118].

b) Geometric Methods: Geometric techniques exploit the geometric interpretations of the first-step parameter estimates to obtain the position of the VLC receiver. In the following, we elaborate on how geometric properties of measurements related to distance (TOA/RSS), angle (AOA), and distance difference (TDOA) can be utilized for localization.

- Distance-based geometric localization: In geometric position estimation, a distance estimate between an LED transmitter and the VLC receiver, which can be derived from TOA and/or RSS measurements, ${ }^{9}$ delineates a sphere around the LED transmitter corresponding to possible locations of the VLC receiver. Based on distance measurements from four (three) LEDs in 3-D (2-D) positioning, the VLC receiver is able to estimate its location as the intersection point of four (three) spheres (circles), a process which is called trilateration.

\footnotetext{
${ }^{8}$ If the receiver can determine the identity of multiple LED transmitters, meaning that the receiver is connected to multiple LEDs or that the receiver is able to decode the signals coming from multiple LEDs, then it selects the one with the largest RSS value as the nearest LED [106].

${ }^{9}$ As mentioned in Section II-B1, an RSS measurement can be translated to a distance estimate only under certain conditions.
}

As an example, for 2-D VLP systems where the height of the VLC receiver is known, consider the following noiseless model for distance measurements between $N_{\mathrm{L}}$ LED transmitters and the VLC receiver:

$$
\begin{aligned}
\left(l_{\mathrm{r}, 1}-l_{\mathrm{t}, 1}^{i}\right)^{2}+\left(l_{\mathrm{r}, 2}-l_{\mathrm{t}, 2}^{i}\right)^{2}= & \left(\hat{d}_{i}^{\text {hor }}\right)^{2}, \\
& i=1,2, \ldots, N_{\mathrm{L}}
\end{aligned}
$$

where $l_{\mathrm{r}, k}$ and $l_{\mathrm{t}, k}^{i}$ denote, respectively, the $k$ th component of the position vector of the VLC receiver and the ith LED transmitter, and $\hat{d}_{i}^{\text {hor }}$ is the horizontal distance measurement between the VLC receiver and the ith LED transmitter. ${ }^{10}$ After some algebraic manipulations, (31) can be expressed as

$$
\mathrm{Ax}=\mathbf{b}
$$

where $\mathbf{x}=\left[l_{\mathrm{r}, 1} l_{\mathrm{r}, 2}\right]$ is the unknown horizontal location of the VLC receiver, and the entries of $\mathbf{A} \in \mathbb{R}^{\left(N_{\mathrm{L}}-1\right) \times 2}$ and $\mathbf{b} \in \mathbb{R}^{\left(N_{\mathrm{L}}-1\right) \times 1}$ are given, respectively, by [44], [45], [59]

$$
\begin{aligned}
A_{i, k}= & l_{\mathrm{t}, k}^{i+1}-l_{\mathrm{t}, k}^{1} \\
b_{i}= & \frac{\left(\hat{d}_{1}^{\text {hor }}\right)^{2}-\left(\hat{d}_{i+1}^{\text {hor }}\right)^{2}+\left(l_{\mathrm{t}, 1}^{i+1}\right)^{2}+\left(l_{\mathrm{t}, 2}^{i+1}\right)^{2}}{2} \\
& -\frac{\left(l_{\mathrm{t}, 1}^{1}\right)^{2}+\left(l_{\mathrm{t}, 2}^{1}\right)^{2}}{2}
\end{aligned}
$$

for $i \in\left\{1,2, \ldots, N_{\mathrm{L}}-1\right\}$ and $k \in\{1,2\}$. In the practical case of noisy distance measurements, the linear least squares (LLS) estimate $\hat{\mathbf{x}}$ of $\mathbf{x}$ can be obtained as [44], [45], [59]

$$
\hat{\mathbf{x}}=\left(\mathbf{A}^{T} \mathbf{A}\right)^{-1} \mathbf{A}^{T} \mathbf{b} .
$$

- Angle-based geometric localization: In addition to distance-based geometric positioning, there also exist localization techniques that leverage the geometric implications of angle measurements to estimate the position of the VLC receiver [55], [60]. The set of possible locations of the VLC receiver based on an AOA measurement from an LED transmitter lies on a straight line passing through the LED. Then, two AOA measurements can be used to specify the location of the VLC receiver as the intersection point of the two lines defined by these measurements in the ideal case. To express the geometric relations in a formal manner, consider a 2-D localization scenario in which the noiseless

${ }^{10} \hat{d}_{i}^{\text {hor }}$ is obtained by $\hat{d}_{i}^{\text {hor }}=\sqrt{\left(\hat{d}_{i}\right)^{2}-h_{i}^{2}}$ where $\hat{d}_{i}$ is the distance measurement between the ith LED transmitter and the VLC receiver and $h_{i}$ is the known height of the transmitter with respect to the receiver. 
AOA estimate $\hat{\varphi}_{i}$ related to the ith LED transmitter is expressed as [60]

$$
\tan \hat{\varphi}_{i}=\frac{l_{\mathrm{r}, 2}-l_{\mathrm{t}, 2}^{i}}{l_{\mathrm{r}, 1}-l_{\mathrm{t}, 1}^{i}}, \quad i=1,2, \ldots, N_{\mathrm{L}} .
$$

Then, based on (36), the following linear relation can be obtained [60]:

$$
\mathbf{A x}=\mathbf{b}
$$

where $\mathbf{x}=\left[l_{\mathrm{r}, 1} l_{\mathrm{r}, 2}\right]$ denotes the $\mathrm{VLC}$ receiver location, and $\mathbf{A} \in \mathbb{R}^{N_{\mathrm{L}} \times 2}$ and $\mathbf{b} \in \mathbb{R}^{N_{\mathrm{L}} \times 1}$ consist of the following elements:

$$
\begin{aligned}
A_{i, 1} & =\sin \hat{\varphi}_{i} \\
A_{i, 2} & =-\cos \hat{\varphi}_{i} \\
b_{i} & =l_{\mathrm{t}, 1}^{i} \sin \hat{\varphi}_{i}-l_{\mathrm{t}, 2}^{i} \cos \hat{\varphi}_{i}
\end{aligned}
$$

for $i \in\left\{1,2, \ldots, N_{\mathrm{L}}\right\}$. Similar to (35), in the case of noisy measurements, the LLS estimate $\hat{\mathbf{x}}$ of the unknown location $\mathbf{x}$ can be calculated as

$$
\hat{\mathbf{x}}=\left(\mathbf{A}^{T} \mathbf{A}\right)^{-1} \mathbf{A}^{T} \mathbf{b} .
$$

- Distance-difference-based geometric localization: In the absence of measurement noise, a TDOA measurement specifies the difference of distances from the VLC receiver to two LED transmitters, and implies that the receiver must be located on a hyperbola the focus of which is the closest LED transmitter (considering a 2-D VLP scenario with a known receiver height) [106]. Hence, it is possible to determine the position of the VLC receiver using two TDOA measurements, which yield a unique intersection point of the two respective hyperbolas under certain conditions [81]. To formulate the TDOA-based localization, the following TDOA measurement model is adopted via (29):

$$
\Delta \tau_{\mathrm{i} 1}=\frac{\left\|\boldsymbol{l}_{\mathrm{r}}-\boldsymbol{l}_{\mathrm{t}}^{\mathrm{i}}\right\|-\left\|\boldsymbol{l}_{\mathrm{r}}-\boldsymbol{l}_{\mathrm{t}}^{1}\right\|}{c}, \quad i=2, \ldots, N_{\mathrm{L}}
$$

where the first LED is selected as the reference. In the presence of noisy TDOA measurements $\Delta \hat{\tau}_{i 1}$ obtained by (28), the unknown location $\boldsymbol{l}_{\mathrm{r}}$ can be estimated by the following nonlinear least squares (NLS) estimator [119]:

$$
\hat{\boldsymbol{l}}_{\mathrm{r}}=\arg \min _{\boldsymbol{l}_{\mathrm{r}}} \sum_{i=2}^{N_{\mathrm{L}}}\left(\Delta \tau_{i 1}-\Delta \hat{\tau}_{i 1}\right)^{2}
$$

where the dependence of $\Delta \tau_{i 1}$ on $\boldsymbol{l}_{\mathrm{r}}$, expressed in (42), is used for finding the optimal location in (43). Apart from the NLS estimator, the TDOAbased geometric techniques involving linear and quadratic equations can also be employed for localization [33]. c) Statistical Methods: Statistical positioning methods make use of the statistical properties of the parameter measurements obtained in the first step to design position estimators. Contrary to geometric techniques, which lack mathematical rigor and depend solely on insights derived from the localization geometry, statistical techniques constitute a methodical way of position estimation and thus can provide asymptotic optimality/performance guarantees [81]. For instance, the ML position estimators in [55], [63], [64], and [102] can asymptotically achieve the CRLB as the SNR increases. In the following, we first present a generic formulation for position estimation based on a statistical measurement model and then introduce statistical estimators employed in synchronous, quasi-synchronous, and asynchronous VLP systems.

- Generic formulation: Consider the following measurements obtained by the VLC receiver in the parameter estimation step (i.e., the first step) of a two-step positioning approach [61]:

$$
\chi=\omega\left(l_{\mathrm{r}}\right)+\zeta
$$

with $\chi=\left[\chi_{1} \ldots \chi_{N_{\mathrm{L}}}\right]^{T}, \boldsymbol{\omega}\left(\boldsymbol{l}_{\mathrm{r}}\right)=\left[\omega_{1}\left(\boldsymbol{l}_{\mathrm{r}}\right) \ldots \omega_{N_{\mathrm{L}}}\left(\boldsymbol{l}_{\mathrm{r}}\right)\right]^{T}$, and $\zeta=\left[\zeta_{1} \ldots \zeta_{\mathrm{N}_{\mathrm{L}}}\right]^{T}$, where $\chi_{\mathrm{i}}$ denotes the first-step parameter measurement corresponding to the ith LED transmitter, $\omega_{i}\left(\boldsymbol{l}_{\mathbf{r}}\right)$ is the true value of the parameter related to the ith LED transmitter, which depends on the location $\boldsymbol{l}_{\mathrm{r}}$ of the VLC receiver, and $\zeta_{i}$ is the noise for the ith measurement. For example, $\omega_{i}\left(\boldsymbol{l}_{\mathrm{r}}\right)$ may represent the TOA parameter in (2), the RSS parameter in (4), the TDOA parameter ${ }^{11}$ in (29), or the AOA parameter in (36). Assuming that the probability density function (PDF) of the noise vector $\zeta$ is given by $f_{\zeta}(\cdot)$, the conditional PDF of the measurement vector $\chi$ given $\boldsymbol{l}_{\mathrm{r}}$ can be expressed as [61]

$$
f_{\chi}\left(\chi \mid \boldsymbol{l}_{\mathrm{r}}\right)=f_{\zeta}\left(\chi-\omega\left(\boldsymbol{l}_{\mathrm{r}}\right)\right)
$$

Based on the availability of prior information on $\boldsymbol{l}_{\mathrm{r}}$, several statistical estimators can be investigated. In the absence of prior information, the ML estimator, which maximizes the likelihood of observations in (45), can be employed to estimate $\boldsymbol{l}_{\mathrm{r}}$ [62]

$$
\hat{\boldsymbol{l}}_{\mathrm{r}}^{\mathrm{ML}}=\arg \max _{\boldsymbol{l}_{\mathrm{r}}} f_{\chi}\left(\chi \mid \boldsymbol{l}_{\mathrm{r}}\right) .
$$

If the prior information on $\boldsymbol{l}_{\mathrm{r}}$ is available, Bayesian estimators can be used for position estimation. Denoting the prior PDF of $\boldsymbol{l}_{\mathrm{r}}$ by $\pi\left(\boldsymbol{l}_{\mathrm{r}}\right)$, the two wellknown Bayesian estimators, namely, the maximum a posteriori probability (MAP) estimator and the

\footnotetext{
${ }^{11}$ In the case of TDOA measurements, the number of measurements that carry information reduces to $N_{L}-1$ since the first LED is selected as the reference for TDOA calculations [see (42)].
} 
minimum mean square error (MMSE) estimator, can be obtained, respectively, as [62]

$$
\begin{aligned}
\hat{\boldsymbol{l}}_{\mathrm{r}}^{\mathrm{MAP}} & =\underset{\boldsymbol{l}_{\mathrm{r}}}{\arg \max _{\chi}} f_{\chi}\left(\chi \mid \boldsymbol{l}_{\mathrm{r}}\right) \pi\left(\boldsymbol{l}_{\mathrm{r}}\right) \\
\hat{l}_{\mathrm{r}}^{\mathrm{MMSE}} & =\mathbb{E}\left\{\boldsymbol{l}_{\mathrm{r}} \mid \chi\right\} .
\end{aligned}
$$

- ML position estimation in synchronous VLP systems: For synchronous VLP systems, both TOA and RSS parameters can be calculated in the first step and employed for position estimation in the second step. First, the asymptotic characteristics of the TOA and RSS estimates are discussed, which provides a basis for designing ML position estimators. Let $\tau \triangleq\left[\tau_{1} \ldots \tau_{N_{L}}\right]^{T}$ and $\hat{\tau} \triangleq\left[\hat{\tau}_{1} \ldots \hat{\tau}_{N_{\mathrm{L}}}\right]^{T}$, where $\tau_{i}$ is the true value of the $i$ th TOA parameter in (2) (with $\Delta_{i}=0$ ) and $\hat{\tau}_{i}$ is the ith TOA estimate in (26). Similarly, let $\alpha \triangleq\left[\alpha_{1} \ldots \alpha_{N_{\mathrm{L}}}\right]^{T}$ and $\hat{\alpha} \triangleq\left[\hat{\alpha}_{1} \ldots \hat{\alpha}_{N_{\mathrm{L}}}\right]^{T}$, where $\alpha_{i}$ and $\hat{\alpha}_{i}$ denote, respectively, the true value of the ith RSS parameter in (4) and the ith RSS estimate in (22). Assuming that $E_{3}^{i}=0$ for $i=1, \ldots, N_{\mathrm{L}}$ [see (13)], the TOA estimates $\hat{\tau}$ and the RSS estimates $\hat{\alpha}$ can be modeled as independent Gaussian random vectors in the high SNR regime (i.e., for $\alpha_{i}^{2} R_{p}^{2} E_{2}^{i} \gg \sigma^{2}$; cf., (1) and (6)) as follows [63]:

$$
\begin{gathered}
\hat{\tau} \sim \mathcal{N}\left(\tau, \Sigma_{\tau}\right) \\
\hat{\alpha} \sim \mathcal{N}\left(\alpha, \Sigma_{\alpha}\right)
\end{gathered}
$$

where

$$
\begin{aligned}
& \Sigma_{\tau}=\operatorname{diag}\left(\left\{\frac{\sigma^{2}}{R_{p}^{2} \alpha_{i}^{2} E_{1}^{i}}\right\}_{i=1}^{N_{\mathrm{L}}}\right) \\
& \Sigma_{\alpha}=\operatorname{diag}\left(\left\{\frac{\sigma^{2}}{R_{p}^{2} E_{2}^{i}}\right\}_{i=1}^{N_{\mathrm{L}}}\right)
\end{aligned}
$$

with $\operatorname{diag}(\cdot)$ denoting the diagonal matrix, $\mathcal{N}(\mu, \Sigma)$ representing the Gaussian distribution with mean vector $\mu$ and covariance matrix $\Sigma$, and $E_{1}^{i}$ and $E_{2}^{i}$ given by (12) and (6), respectively. It is noted that (49) and (50) result from the asymptotic unbiasedness and efficiency properties of ML estimation [62]. Next, from (49) and (50), the ML estimator for $\boldsymbol{l}_{\mathrm{r}}$ based on the first-step TOA and RSS estimates $\left\{\hat{\tau}_{i}, \hat{\alpha}_{i}\right\}_{i=1}^{N_{\mathrm{L}}}$ is obtained as [63]

$$
\begin{aligned}
& \hat{\boldsymbol{l}}_{\mathrm{r}}^{\mathrm{TS}, \mathrm{syn}}=\arg \min _{\boldsymbol{l}_{r}} \sum_{i=1}^{N_{\mathrm{L}}}\left(E_{1}^{i} \alpha_{i}^{2}\left(\hat{\tau}_{i}-\tau_{i}\right)^{2}+E_{2}^{i}\left(\hat{\alpha}_{i}-\alpha_{i}\right)^{2}\right) \\
& -\frac{2 \sigma^{2}}{R_{p}^{2}} \sum_{i=1}^{N_{\mathrm{L}}} \log \alpha_{i}
\end{aligned}
$$

where the optimal $\boldsymbol{l}_{\mathrm{r}}$ is searched via the relations of $\tau_{i}$ and $\alpha_{i}$ with $\boldsymbol{l}_{\mathrm{r}}$, as defined, respectively, in (2) (with $\Delta_{i}=0$ ) and (4). In the high SNR regime, the last term in (53) becomes negligible as compared to the others and $\alpha_{i}$ in the first term is approximately equal to $\hat{\alpha}_{i}$ via (50). Hence, the estimator in (53) can be simplified to [63]

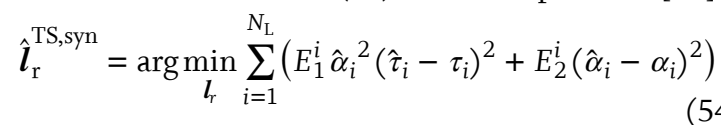

which is an NLS estimator. It is shown in [63, Prop. 2] that the NLS estimator in (54) is asymptotically optimal, i.e., attains the CRLB in (10) at high SNRs.

- ML position estimation in quasi-synchronous VLP systems: Since the LED transmitters and the VLC receiver are not synchronized in quasi-synchronous VLP systems, TOA measurements cannot directly be utilized in the second step of a two-step estimator. However, by virtue of synchronization among the LED transmitters, TDOA measurements can be employed along with RSS measurements to design an ML estimator for $\boldsymbol{l}_{\mathrm{r}}$. Choosing the first LED as the reference, let $\Delta \tau \triangleq\left[\Delta \tau_{21} \ldots \Delta \tau_{N_{\mathrm{L}} 1}\right]^{T}$ and $\Delta \hat{\tau} \triangleq\left[\Delta \hat{\tau}_{21} \ldots \Delta \hat{\tau}_{N_{\mathrm{L}} 1}\right]^{T}$, where $\Delta \tau_{i 1}$ and $\Delta \hat{\tau}_{i 1}$ denote, respectively, the true value of the ith TDOA parameter in (42) and the $i$ th TDOA estimate in (28). Then, for $E_{3}^{i}=0, i=$ $1, \ldots, N_{\mathrm{L}}$, the TDOA and RSS measurement vectors are independent and distributed asymptotically (at high SNRs) as [94]

$$
\begin{gathered}
\Delta \hat{\tau} \sim \mathcal{N}\left(\Delta \tau, \Sigma_{\Delta \tau}\right) \\
\hat{\alpha} \sim \mathcal{N}\left(\alpha, \Sigma_{\alpha}\right)
\end{gathered}
$$

where $\Sigma_{\alpha}$ is as in (52) and

$$
\Sigma_{\Delta \tau}=\frac{\sigma^{2}}{R_{p}^{2} \alpha_{1}^{2} E_{1}^{1}} \mathbf{1}+\operatorname{diag}\left(\left\{\frac{\sigma^{2}}{R_{p}^{2} \alpha_{i}^{2} E_{1}^{i}}\right\}_{i=2}^{N_{\mathrm{L}}}\right)
$$

with 1 denoting the all-ones matrix. Then, the ML estimate of $\boldsymbol{l}_{\mathrm{r}}$ based on the TDOA estimates $\Delta \hat{\boldsymbol{\tau}}$ and the RSS estimates $\hat{\alpha}$ can be written as [94]

$\hat{\boldsymbol{l}}_{\mathrm{r}}^{\mathrm{TS}, \mathrm{qsy}}=\arg \min _{\boldsymbol{l}_{\mathrm{r}}}(\hat{\boldsymbol{v}}-\boldsymbol{v})^{\mathrm{T}} \Sigma^{-1}(\hat{v}-\boldsymbol{v})+\log \left|\Sigma_{\Delta \tau}\right|$

where $\hat{\boldsymbol{v}} \triangleq\left[\Delta \hat{\tau}^{T} \hat{\boldsymbol{\alpha}}^{T}\right]^{T}, \boldsymbol{v} \triangleq\left[\boldsymbol{\Delta} \tau^{T} \boldsymbol{\alpha}^{T}\right]^{T}$, and $\Sigma \triangleq \operatorname{Diag}\left(\Sigma_{\boldsymbol{}}\right.$, $\Sigma_{\alpha}$ ) with $\operatorname{Diag}(\cdot)$ denoting the block diagonal matrix. The cost function in (58) depends on the unknown location $\boldsymbol{l}_{\mathrm{r}}$ via $v, \Sigma_{\Delta \tau}$, and $\Sigma$.

- ML position estimation in asynchronous VLP systems: Since time-based information cannot be obtained in asynchronous VLP systems, only the RSS measurements can be utilized for the second step of the twostep position estimation. Then, the ML estimator for $\boldsymbol{l}_{\mathrm{r}}$ based on the RSS measurements can be written as [63]

$$
\hat{\boldsymbol{l}}_{\mathrm{r}}^{\mathrm{TS} \text {,asy }}=\arg \min _{\boldsymbol{l}_{\mathrm{r}}} \sum_{i=1}^{N_{\mathrm{L}}} w_{i}\left(\hat{\alpha}_{i}-\alpha_{i}\right)^{2}
$$

where $\alpha_{i}$ is the true value of the RSS parameter associated with the ith LED transmitter and depends on $\boldsymbol{l}_{\mathrm{r}}$ 
via (4), $\hat{\alpha}_{i}$ is the RSS estimate for the ith LED transmitter, which is obtained by (22), and the weighting coefficients $w_{i}$ are defined as [63]

$$
w_{i}=E_{2}^{i}-\frac{\left(E_{3}^{i}\right)^{2}}{E_{1}^{i}} \text {. }
$$

The proposed weighting coefficient in (60) is determined according to the reliability of each RSS estimate. Therefore, $w_{i}$ is inversely proportional to the CRLB for estimating $\alpha_{i}$ from the received signal $r_{i}(t)$, as observed from (24). As demonstrated in [63, Prop. 4], the two-step estimator in (59) is equivalent to the direct estimator in (8) for $E_{3}^{i}=0$. Thus, the two-step estimator is the optimal ML estimator in asynchronous VLP systems for practical pulse shapes.

d) Fingerprinting/Mapping Methods: A fingerprinting method for position estimation generally consists of two phases, namely, the offline phase and the online phase. In the offline phase, a database is constructed by gathering measurements over a grid of reference points in an indoor environment [65], [66]. Each entry of the database stores the location of the specified reference point and the parameter estimates (e.g., RSS, TOA, TDOA, AOA, or a combination of them) associated with the LED transmitters obtained at that location [65], [66]. In the online phase, the vector of parameter estimates measured by the VLC receiver is compared with the database to decide on the location of the receiver according to a proximity measure between the online measurement vector and the offline prestored database [17], [65], [66]. In addition, a decision rule that maps the online feature/measurement vector to a location in the scene can be devised using machine learning techniques, such as $k$ nearest neighbor $(k-N N)$, neural networks, and random forest [120]. Hence, based on the training database containing offline parameter measurements, a fingerprinting/mapping method can learn a classifier through which online measurements are mapped to corresponding locations in the room.

One of the most common parameters employed in fingerprinting methods for VLP systems is the RSS parameter [28], [65], [66], [120]-[126] since it does not require synchronization as in the case of TOA and TDOA measurements, and it can simply be measured via a single PD at the receiver as opposed to AOA measurements. Another parameter that can be preferred for fingerprinting is the extinction ratio, which refers to the difference between the received powers corresponding to bit- 0 and bit- 1 transmissions [127].

Given a comprehensive offline database with densely placed reference points, fingerprinting/mapping techniques are capable of producing more accurate location estimates as compared to geometric positioning methods [65]. The primary disadvantage of fingerprinting techniques over geometric, statistical, and proximity-based position estimation methods is that they involve the process of building and maintaining an offline training database, which aggravates the computational complexity in dynamic scenes [81].

\section{COOPERATIVE VISIBLE LIGHT POSITIONING}

As shown by numerous studies in the literature, cooperation among target nodes (i.e., nodes with unknown positions) can enhance performance of RF-based localization systems [34], [70]-[72], [128]-[133]. Similarly, cooperation among VLC units in a VLP system can be useful for achieving improved localization performance compared to the case in which VLC units gather signals only from the LED transmitters at known positions. In this section, we investigate a new VLP system architecture that facilitates communications among VLC units for the purpose of cooperative localization and discuss an iterative algorithm based on gradient projections to estimate locations of VLC units.

\section{A. System Model}

In a cooperative VLP system, there exist $L_{0}$ LED transmitters with known locations and orientations (i.e., anchor/ reference nodes), and $N_{V}$ VLC units that are to be located (i.e., agent/target nodes). The location of the $\ell$ th LED transmitter is denoted by $\mathrm{y}_{\ell}$ and its orientation vector is given by $\mathbf{n}_{T, \ell}^{(0)}$ for $\ell \in\left\{1, \ldots, L_{0}\right\}$. Each VLC unit not only receives signals from the LED transmitters at known locations but also communicates with other VLC units in the system for cooperation purposes [67]. Therefore, VLC units consist of both LEDs and PDs; namely, there exist $L_{i}$ LEDs and $K_{i}$ PDs at the $i$ th VLC unit for $i \in\left\{1, \ldots, N_{V}\right\}$. The unknown location of the $i$ th VLC unit is represented by $\mathbf{x}_{i}$, where $i \in\left\{1, \ldots, N_{V}\right\}$. For the kth PD at the ith VLC unit, the location is denoted by $\mathbf{x}_{i}+\mathbf{a}_{i, k}$ and the orientation vector is given by $\mathbf{n}_{R, k}^{(i)}$, where $k \in\left\{1, \ldots, K_{i}\right\}$. Similarly, for the $\ell$ th LED at the ith VLC unit, the location is represented by $\mathbf{x}_{i}+\mathbf{b}_{i, \ell}$ and the orientation vector is denoted by $\mathbf{n}_{T, \ell}^{(i)}$, where $\ell \in\left\{1, \ldots, L_{i}\right\}$. The displacement vectors $\mathbf{a}_{i, k}$ 's and $\mathbf{b}_{i, \ell}$ 's are known design parameters of the VLC units [67]. In addition, the orientation vectors for the LEDs and PDs at the VLC units are assumed to be known since they can be determined by the VLC unit design and/or via auxiliary sensors such as gyroscopes [55], [67], [134]. To distinguish the LED transmitters at known locations from the LEDs at the VLC units, the former are called as the LEDs on the ceiling in the remainder of this section (see Fig. 4).

In the cooperative VLP system, each PD communicates with a subset of all the LEDs in the network. For this reason, the following connectivity sets are defined to specify the connections between the PDs and the LEDs [67]:

$$
\begin{aligned}
S_{k}^{(i, j)}=\left\{\ell \in\left\{1, \ldots, L_{i}\right\} \mid \ell \text { th LED of } i\right. \text { thVLC unit is } \\
\text { connected to } k \text { th PD of } j \text { th VLC unit }\},
\end{aligned}
$$$$
k \in\left\{1, \ldots, K_{j}\right\}, \quad i \in\left\{0,1, \ldots, N_{V}\right\}, j \in\left\{1, \ldots, N_{V}\right\} .
$$ 


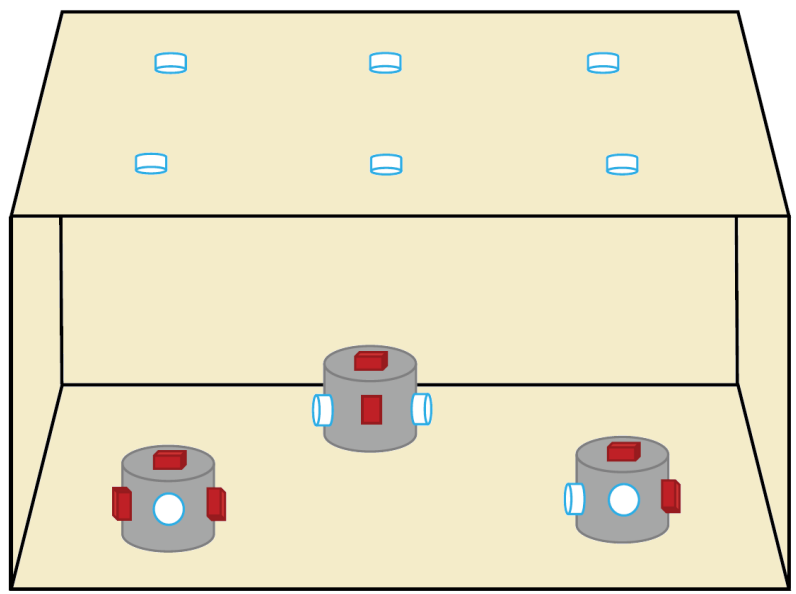

Fig. 4.Illustration of a cooperative VLP system with three VLC units (e.g., robots). The white cylinders on the ceiling and at the VLC units represent the LEDs, and the red rectangular prisms denote the PDs.

Namely, $S_{k}^{(i, j)}$ represents the set of LEDs at the ith VLC unit that are connected to the $k$ th PD at the jth VLC unit. It is noted that the LEDs on the ceiling are considered as the zeroth VLC unit in order to develop a unified formulation. In other words, "VLC unit 0" is a hypothetical VLC unit with $L_{0}$ LEDs (at known locations) and zero PDs. Hence, index $i$ starts from zero in (61).

We consider a scenario in which RSS measurements performed by the PDs are employed for estimating the unknown locations of the VLC units, i.e., $\mathbf{x}_{1}, \ldots, \mathbf{x}_{N_{V}}$. Let $P_{\ell, k}^{(i, j)}$ represent the RSS measurement at the $k$ th PD of the $j$ th VLC unit due to the transmission from the $\ell$ th LED at the ith VLC unit. From the Lambertian formula [93], $P_{\ell, k}^{(i, j)}$ can be stated as follows:

$$
\begin{aligned}
P_{\ell, k}^{(i, j)} & =\frac{m_{\ell}^{(i)}+1}{2 \pi} P_{T, \ell}^{(i)} \cos _{\ell}^{m_{\ell}^{(i)}}\left(\phi_{\ell, k}^{(i, j)}\right) \cos \left(\theta_{\ell, k}^{(i, j)}\right) \frac{A_{k}^{(j)}}{\left(d_{\ell, k}^{(i, j)}\right)^{2}} \\
& +\eta_{\ell, k}^{(i, j)}
\end{aligned}
$$

for $j \in\left\{1, \ldots, N_{V}\right\}, k \in\left\{1, \ldots, K_{j}\right\}, i \in\left\{0,1, \ldots, N_{V}\right\} \backslash j$, and $\ell \in$ $S_{k}^{(i, j)}$, where the distance $d_{\ell, k}^{(i, j)}$ is given by

$$
d_{\ell, k}^{(i, j)}=\left\|\mathbf{d}_{\ell, k}^{(i, j)}\right\|
$$

with

$$
\mathbf{d}_{\ell, k}^{(i, j)} \triangleq \begin{cases}\mathbf{x}_{j}+\mathbf{a}_{j, k}-\mathbf{x}_{i}-\mathbf{b}_{i, \ell}, & \text { if } i \neq 0 \\ \mathbf{x}_{j}+\mathbf{a}_{j, k}-\mathbf{y}_{\ell}, & \text { if } i=0 .\end{cases}
$$

In (62), $m_{\ell}^{(i)}$ is the Lambertian order for the $\ell$ th LED at the ith VLC unit, $A_{k}^{(j)}$ is the area of the kth PD at the $j$ th VLC unit, $P_{T, \ell}^{(i)}$ is the transmit power of the $\ell$ th LED at the ith VLC unit, $\phi_{\ell, k}^{(i, j)}$ is the irradiation angle at the $\ell$ th LED at the ith VLC unit with respect to the $k$ th PD at the jth VLC unit, and $\theta_{\ell, k}^{(i, j)}$ is the incidence angle for the kth PD at the jth VLC unit related to the $\ell$ th LED at the ith VLC unit. The noise component $\eta_{\ell, k}^{(i, j)}$ is modeled by a random variable with a generic $\operatorname{PDF} f_{\eta}^{(j, k)}(\cdot)$. Supposing the use of a certain multiplexing scheme (e.g., time-division multiplexing among the LEDs at the same VLC unit and on the ceiling, and frequency-division multiplexing among the LEDs at different VLC units or on the ceiling), $\eta_{\ell, k}^{(i, j)}$, s are assumed to be independent for all different $(j, k)$ pairs and for all $\ell$ and $i$ [67], [104], [106]. From (63) and (64), the RSS measurements in (62) can also be expressed as follows [67]:

$$
P_{\ell, k}^{(i, j)}=\alpha_{\ell, k}^{(i, j)}+\eta_{\ell, k}^{(i, j)}
$$

where

$\alpha_{\ell, k}^{(i, j)} \triangleq-\frac{m_{\ell}^{(i)}+1}{2 \pi} P_{T, \ell}^{(i)} A_{k}^{(j)} \frac{\left(\left(\mathbf{d}_{\ell, k}^{(i, j)}\right)^{T} \mathbf{n}_{T, \ell}^{(i)}\right)^{m_{\ell}^{(i)}}\left(\mathbf{d}_{\ell, k}^{(i, j)}\right)^{T} \mathbf{n}_{R, k}^{(j)}}{\left\|\mathbf{d}_{\ell, k}^{(i, j)}\right\|^{m_{\ell}^{(i)}+3}}$.

Remark: The proposed system model for cooperative VLP applications relies on one-shot position estimation that utilizes the parameter values of a given configuration to estimate the locations of VLC units at a given instant. In VLP scenarios with mobile VLC units, certain parameters, such as locations and orientations of VLC units, are highly time varying and need to be updated at each decision/estimation step. For instance, the displacement vectors $\mathbf{a}_{i, k}$ and $\mathbf{b}_{i, \ell}$ depend on the orientation vectors $\mathbf{n}_{R, k}^{(i)}$ and $\mathbf{n}_{T, \ell}^{(i)}$, respectively. Hence, the values of $\mathbf{a}_{i, k}$ and $\mathbf{b}_{i, \ell}$ can be updated at each time step using the new values of $\mathbf{n}_{R, k}^{(i)}$ and $\mathbf{n}_{T, \ell}^{(i)}$ (e.g., via rotation matrices). For the proposed localization framework, it is assumed that the current values of all localization related parameters are known and thus can be employed for position estimation at the current time step.

\section{B. Localization Algorithms}

Let the vector of unknown parameters be represented as $\mathbf{x} \triangleq\left[\mathbf{x}_{1}^{T} \ldots \mathbf{x}_{N_{V}}^{T}\right]^{T}$, which has a size of $3 N_{V} \times 1$. The aim is to estimate the elements of $\mathbf{x}$ based on the RSS measurements in (62) [equivalently, in (65)]. To this aim, various approaches can be considered, as discussed in the following.

1) Centralized Approach:In this approach, all the RSS measurements are processed at a central unit to estimate the locations of the VLC units jointly. Let $\mathbf{P}$ denote a vector consisting of all the measurements in (65). Namely, the elements of $\mathbf{P}$ are expressed as follows:

$$
\left\{\left\{\left\{\left\{P_{\ell, k}^{(i, j)}\right\}_{\ell \in S_{k}^{(i, j)}}\right\}_{i \in\left\{0,1, \ldots, N_{V}\right\} \backslash\{j\}}\right\}_{k \in\left\{1, \ldots, K_{j}\right\}}\right\}_{j \in\left\{1, \ldots, N_{V}\right\}}
$$

Then, the ML estimate of $\mathbf{x}$ based on $\mathbf{P}$ is given by [62]

$$
\widehat{\mathbf{x}}_{\mathrm{ML}}=\arg \max _{\mathbf{x}} f(\mathbf{P} \mid \mathbf{x})
$$

where $f(\mathbf{P} \mid \mathbf{x})$ denotes the conditional PDF of $\mathbf{P}$ given $\mathbf{x}$, i.e., the likelihood function. For example, if the noise PDF $f_{\eta}^{(j, k)}(\cdot)$ follows a Gaussian distribution with zero mean and a variance of $\sigma_{j, k}^{2}$, then the ML estimate in (67) can be obtained from (65) as follows [67]:

$$
\widehat{\mathbf{x}}_{\mathrm{ML}}=\arg \min _{\mathbf{x}} \sum_{j=1}^{N_{V}} \sum_{k=1}^{K_{j}} \frac{1}{\sigma_{j, k}^{2}} \sum_{i=0, i \neq j}^{N_{V}} \sum_{\ell \in S_{k}^{(i, j)}}\left(P_{\ell, k}^{(i, j)}-\alpha_{\ell, k}^{(i, j)}\right)^{2}
$$


where $\alpha_{\ell, k}^{(i, j)}$ is calculated from (66) via (64).

The problem in (67) has high computational complexity since it requires a search over a $3 N_{V}$-dimensional space. Therefore, it may not be employed in practical applications. Hence, decentralized approaches with lower complexity can be considered, as discussed next.

2) Decentralized Approach: Since the objective function in (67) is, in general, nonconcave with respect to the vector of unknown locations $\mathbf{x}$ via (64) and (66) [e.g., nonconvexity of the objective function in (68)], the problem in (67) cannot be solved to global optimality using standart convex optimization methods. In addition, obtaining globally optimal solutions of (67) via global optimization tools may lead to an excessive computational burden, especially as the number of VLC units increases [68]. Therefore, we formulate the problem of cooperative localization as a feasibility problem and propose a lowcomplexity iterative algorithm that can be implemented in a decentralized manner to solve the feasibility problem [68]. In the following, we first present the problem formulation and then introduce the proposed cooperative localization algorithm [68].

a) Formulation of Feasibility Problem: In feasibility problems, the aim is to find a point that satisfies certain constraints without having to optimize an objective function, as opposed to optimization problems [135]. Hence, the computational complexity of feasibility-seeking techniques can be significantly lower than that of optimization methods, which makes feasibility modeling an attractive approach for dealing with nonconvex problems. In this part, we present the formulation of the feasibility problem that is intended to approximate the problem of cooperative localization as defined by the ML estimator in (67).

Based on the measurement model in (65), an RSS observation at a PD can be modeled as

$$
\hat{P}_{r}=P_{r}+\eta
$$

where $P_{r}$ denotes the true value of the RSS parameter [as in (66)] and $\eta$ is the measurement noise. To facilitate the feasibility-based formulation, it is assumed that the noise PDF $f_{\eta}(\cdot)$ satisfies $f_{\eta}(\bar{\eta})=0 \forall \bar{\eta}>0$, i.e., the RSS measurement errors are negative $\left(\hat{P}_{r} \leq P_{r}\right){ }^{12}$ Then, using the Lambertian model in (66), a generic Lambertian function

\footnotetext{
${ }^{12}$ We can always subtract a constant value from the obtained RSS measurement to satisfy the assumption of negative measurement noise [136], [137]. For PDFs having finite support, this value can be chosen as $\hat{\eta}=\sup \left\{\bar{\eta} \in \mathbb{R} \mid f_{\eta}(\bar{\eta})>0\right\}$. For PDFs having infinite support, such as Gaussian, we can set $\hat{\eta}=\inf \left\{\bar{\eta} \in \mathbb{R} \mid \int_{-\infty}^{\bar{\eta}} f_{\eta}(\epsilon) d \epsilon \geq 1-\bar{\varpi}\right\}$ for some small probability $\varpi$ to approximately satisfy the assumption. In terms of convergence properties, Algorithm 1, which is proposed later in this section, has a theoretical convergence guarantee under the assumption of negative errors (see [68, Sec. V] for convergence analysis). Although no convergence guarantee can be provided for Algorithm 1 in the case of both positive and negative errors (e.g., Gaussian noise), numerical simulations reveal that the iterations generated by Algorithm 1 are able to converge to true locations asymptotically as the SNR increases.
}

$g: \mathrm{R}^{D} \rightarrow \mathrm{R}$ with respect to the unknown PD location $\mathbf{x} \in \mathbb{R}^{D}$ satisfies $^{13}$

$$
g\left(\mathbf{x} ; \mathbf{y}, \mathbf{n}_{T}, \mathbf{n}_{R}, m, \gamma\right) \leq 0
$$

where

$g\left(\mathbf{x} ; \mathbf{y}, \mathbf{n}_{T}, \mathbf{n}_{R}, m, \gamma\right) \triangleq \gamma-\frac{\left[(\mathbf{x}-\mathbf{y})^{T} \mathbf{n}_{T}\right]^{m}(\mathbf{y}-\mathbf{x})^{T} \mathbf{n}_{R}}{\|\mathbf{x}-\mathbf{y}\|^{m+3}}$

and $\mathbf{y}$ denotes the location of the LED, $\mathbf{n}_{T}$ and $\mathbf{n}_{R}$ are the orientation vectors of the LED and PD, respectively, $m$ is the Lambertian order of the LED, and $\gamma$ is defined as $\gamma=\left(\hat{P}_{r} / P_{t}\right)$ $(2 \pi /((m+1) A))$ with $P_{t}$ and $A$ denoting, respectively, the transmit power of the LED and the area of the PD. Invoking the negative error assumption and using (70), the location of the PD belongs to the following feasible set (referred to as the Lambertian set):

$$
\mathcal{L}=\left\{\mathbf{x} \in \mathbb{R}^{D} \mid g\left(\mathbf{x} ; \mathbf{y}, \mathbf{n}_{T}, \mathbf{n}_{R}, m, \gamma\right) \leq 0\right\} .
$$

Regarding communications between the LEDs on the ceiling and the VLC units, the Lambertian set for kth PD of the jth VLC unit based on the signal emitted by the $\ell$ th LED on the ceiling for $\ell \in S_{k}^{(0, j)}$ is given by (referred to as the noncooperative Lambertian sets)

$$
\mathcal{C}_{\ell, k}^{(0, j)}=\left\{\mathbf{z} \in \mathbb{R}^{D} \mid \tilde{g}_{\ell, k}^{(j)}(\mathbf{z}) \leq 0\right\}
$$

where $\widetilde{g}_{\ell, k}^{(j)}(\mathbf{z})$ is defined as

$$
\tilde{g}_{\ell, k}^{(j)}(\mathbf{z}) \triangleq g\left(\mathbf{z} ; \mathbf{y}_{\ell}-\mathbf{a}_{j, k}, \mathbf{n}_{T, \ell}^{(0)}, \mathbf{n}_{R, k}^{(j)}, m_{\ell}^{(0)}, \gamma_{\ell, k}^{(0, j)}\right)
$$

and $\gamma_{\ell, k}^{(i, j)}=\left(P_{\ell, k}^{(i, j)} / P_{T, \ell}^{(i)}\right)\left(2 \pi /\left(\left(m_{\ell}^{(i)}+1\right) A_{k}^{(j)}\right)\right)$ for $i \in\{0,1, \ldots$, $\left.N_{V}\right\}$ via (65) and (66). Similarly, regarding communications among the VLC units, the Lambertian set for the $k$ th PD of the jth VLC unit based on the signal emitted by the $\ell$ th LED of the ith VLC unit for $\ell \in S_{k}^{(i, j)}$ is given by (referred to as the cooperative Lambertian sets)

$$
\mathcal{C}_{\ell, k}^{(i, j)}=\left\{\mathbf{z} \in \mathbb{R}^{D} \mid g_{\ell, k}^{(i, j)}\left(\mathbf{z}, \mathbf{x}_{i}\right) \leq 0\right\}
$$

for $i \in\left\{1,2, \ldots, N_{V}\right\}$, where $g_{\ell, k}^{(i, j)}\left(\mathbf{z}, \mathbf{x}_{i}\right)$ is defined as

$$
g_{\ell, k}^{(i, j)}\left(\mathbf{z}, \mathbf{x}_{i}\right) \triangleq g\left(\mathbf{z} ; \mathbf{x}_{i}+\mathbf{b}_{i, \ell}-\mathbf{a}_{j, k}, \mathbf{n}_{T, \ell}^{(i)}, \mathbf{n}_{R, k}^{(j)}, m_{\ell}^{(i)}, \gamma_{\ell, k}^{(i, j)}\right) .
$$

Then, the problem of cooperative localization in VLP systems is equivalent to identifying a point inside the intersection of Lambertian sets as defined in (73) and (75). Assuming that the Lambertian function in (71) is quasi-convex, ${ }^{14}$ the

${ }^{13} \mathrm{D}$ is the dimension of localization in the cooperative VLP scenario. For example, when the height of the VLC receiver is known as in [47], [48], [100], and [101], 2-D localization is performed, i.e., $D=2$. However, in general, $D=3$.

${ }^{14}$ For the case of a known PD height and perpendicular LED orientation, i.e., $x_{3}$ is known and $\mathbf{n}_{T}=\left[\begin{array}{lll}0 & 0 & -1\end{array}\right]^{T}$ in $(71)$, the Lambertian function in (71) is quasi-convex [68]. For the general case in which the LED orientation is arbitrary and/or the height of the PD is unknown, we can obtain a quasi-convex approximation of the Lambertian function in (71) [68]. 
quasi-convex feasibility problem (QFP) can be formulated as follows [138], [139].

Problem 1. Let $\mathbf{x} \triangleq\left(\mathbf{x}_{1}, \ldots, \mathbf{x}_{N_{V}}\right)$. The feasibility problem for cooperative localization of VLC units is expressed as

$$
\begin{aligned}
\text { find } & \mathbf{x} \in \mathbb{R}^{D N_{V}} \\
\text { subject to } & \mathbf{x}_{j} \in \Upsilon_{j}, j=1, \ldots, N_{V}
\end{aligned}
$$

where

$$
\Upsilon_{j}=\bigcap_{k=1}^{K_{j}} \bigcap_{i=0}^{N_{V}} \bigcap_{\ell \in S_{k}^{(i, j)}} \mathcal{C}_{\ell, k}^{(i, j)}
$$

with $\mathcal{C}_{\ell, k}^{(i, j)}$ being given by $(73)$ and (75).

QFPs constitute a class of feasibility problems in which the functions characterizing the constraint sets [e.g., the Lambertian function in (71) and the associated constraint set in (72)] are quasi-convex [139]. In the next part, an iterative decentralized algorithm is introduced to solve the QFP in (77). To gain an intuition on the geometry of Problem 1, a noncooperative VLP system and its cooperative version are illustrated along with the corresponding Lambertian sets in Fig. 5.

b) Decentralized Algorithm: In this part, we present a decentralized algorithm based on iterative gradient projections to solve Problem 1. The motivation behind the use of gradient projections is to reach the intersection region of the constraint sets $\mathcal{C}_{\ell, k}^{(i, j)}$ in (78) by moving in the opposite

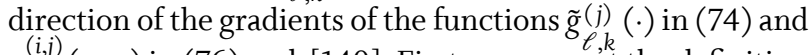
$g_{\ell, k}^{(i, j)}\left(\cdot, \mathbf{x}_{i}\right)$ in (76) and [140]. First, we present the definition of the gradient projection operator.

Definition 1: The gradient projection operator $G_{f}^{\lambda}$ : $\mathbb{R}^{D} \rightarrow \mathbb{R}^{D}$ onto the zero sublevel set of a continuously differentiable function $f: \mathbb{R}^{D} \rightarrow \mathbb{R}$ is given by $[141]$

$$
G_{f}^{\lambda}(\mathbf{x})=\mathbf{x}-\lambda \frac{f^{+}(\mathbf{x})}{\|\nabla f(\mathbf{x})\|^{2}} \nabla f(\mathbf{x})
$$

where $\lambda$ is the relaxation parameter, $\nabla$ is the gradient operator, and $f^{+}(\mathbf{x})$ denotes the positive part, i.e., $f^{+}(\mathbf{x})=\max \{0, f(\mathbf{x})\}$.

Based on Definition 1, we present the proposed algorithm, namely, the cooperative gradient projections (CGP), in Algorithm 1.

The algorithm consists of the following three phases, which are executed for each VLC unit (say, the jth VLC unit) either in parallel or in a sequential manner.

- Projection onto intersection of halfspaces: In order to keep the $n$th iterate $\mathbf{x}_{j}^{(n)}$ inside the region where the noncooperative Lambertian functions defined in (74) are all quasi-convex, $\mathbf{x}_{j}^{(n)}$ is projected onto the intersection of the halfspaces in (82), which are derived from the noncooperative Lambertian sets. ${ }^{15}$ The

\footnotetext{
${ }^{15}$ The reader is referred to $[68$, Sec. III and IV] for a thorough discussion.See [68, Algorithm 1] for the definition of $P_{\Gamma_{j}}(\cdot)$ in (80).
}

\section{Algorithm 1: Cooperative Gradient Projections (CGP)}

Initialization: Choose an arbitrary initial point $\left(\mathbf{x}_{1}^{(0)}, \ldots, \mathbf{x}_{N_{V}}^{(0)}\right) \epsilon$ $\mathbb{R}^{D N_{V}}$

Iterative Step: Given the $n$th iterate $\left(\mathbf{x}_{1}^{(n)}, \ldots, \mathbf{x}_{N_{V}}^{(n)}\right) \in \mathbb{R}^{D N_{V}}$

for $j=1, \ldots, N_{V}$ do

Projection Onto Intersection of Halfspaces $\Gamma_{j}$ by [68, Algorithm 1]:

$$
\tilde{\mathbf{x}}_{j}^{(n)}=P_{\Gamma_{j}}\left(\mathbf{x}_{j}^{(n)}\right)
$$

where the intersection of halfspaces is given by

$$
\Gamma_{j}=\bigcap_{k=1}^{K_{j}} \bigcap_{\ell \in S_{k}^{(0, j)}} \tilde{\Omega}_{\ell, k}^{(j)}
$$

with

$$
\tilde{\Omega}_{\ell, k}^{(j)} \triangleq\left\{\mathbf{x} \in \mathbb{R}^{D} \mid\left(\mathbf{y}_{\ell}-\mathbf{a}_{j, k}-\mathbf{x}\right)^{T} \mathbf{n}_{R, k}^{(j)} \geq 0\right\} .
$$

Parallel Projection Onto Lambertian Sets:

$$
\begin{aligned}
\mathbf{x}_{j}^{(n+1)}=\sum_{k=1}^{K_{j}} & {\left[\sum_{\ell \in S_{k}^{(0, j)}} \tilde{\kappa}_{\ell, k}^{(j)} G_{\tilde{g}_{\ell, k}^{(j)}}^{\lambda_{j}^{(n)}}\left(\tilde{\mathbf{x}}_{j}^{(n)}\right)\right.} \\
& \left.+\sum_{i=1, i \neq j}^{N_{V}} \sum_{\ell \in S_{k}^{(i, j)}} \kappa_{\ell, k}^{(i, j)} G_{g_{\ell, k}^{(i, j)}\left(\cdot, \mathbf{x}_{i}^{(\hat{n})}\right)}^{\lambda^{(n)}}\left(\tilde{\mathbf{x}}_{j}^{(n)}\right)\right]
\end{aligned}
$$

where $\hat{n}=n$ for $i>j, \hat{n}=n+1$ for $i<j$ and the weights satisfy

$$
\sum_{k=1}^{K_{j}}\left(\sum_{\ell \in S_{k}^{(0, j)}} \tilde{\kappa}_{\ell, k}^{(j)}+\sum_{i=1, i \neq j}^{N_{V}} \sum_{\ell \in S_{k}^{(i, j)}} \kappa_{\ell, k}^{(i, j)}\right)=1
$$

and $\tilde{\kappa}_{\ell, k}^{(j)} \geq 0, \kappa_{\ell, k}^{(i, j)} \geq 0, \forall i, \ell, k$.

end for

Stopping Criterion: $\sum_{j=1}^{N_{V}}\left\|\mathbf{x}_{j}^{(n+1)}-\mathbf{x}_{j}^{(n)}\right\|^{2}<\delta$ for some $\delta>0$.

Relaxation Parameters: The relaxation parameters $\lambda_{j}^{(n)}$ are updated using the Armijo rule in [68, Algorithm 2].

quasi-convexity of a Lambertian function guarantees that the gradient projection operator forces the iterates to get closer to the Lambertian set associated with that function.

- Parallel projection onto Lambertian sets: In this step, the current point $\widetilde{\mathbf{x}}_{j}^{(n)}$, which is the output of the previous step, is projected onto each noncooperative and cooperative Lambertian set via the gradient projection operator in (79). Then, the resulting projections are weighted in (83) to obtain the next iterate $\mathbf{x}_{j}^{(n+1)}$. The cooperative Lambertian sets as defined in (75) are constructed from the most recent position information of the other VLC units [i.e., $\mathbf{x}_{i}^{(n)}$ or $\mathbf{x}_{i}^{(n+1)}$ for $i \neq j$ depending on the order of position updates, as shown in (83)].

- Updating relaxation parameters: The relaxation parameters $\lambda_{j}^{(n)}$ for the $j$ th VLC unit at the $n$th iteration, which are used in the gradient projection operator, are updated using the Armijo step size selection rule in [68, Algorithm 2].

We note that Algorithm 1 can be implemented in a decentralized manner via a gossip-like procedure among the VLC 


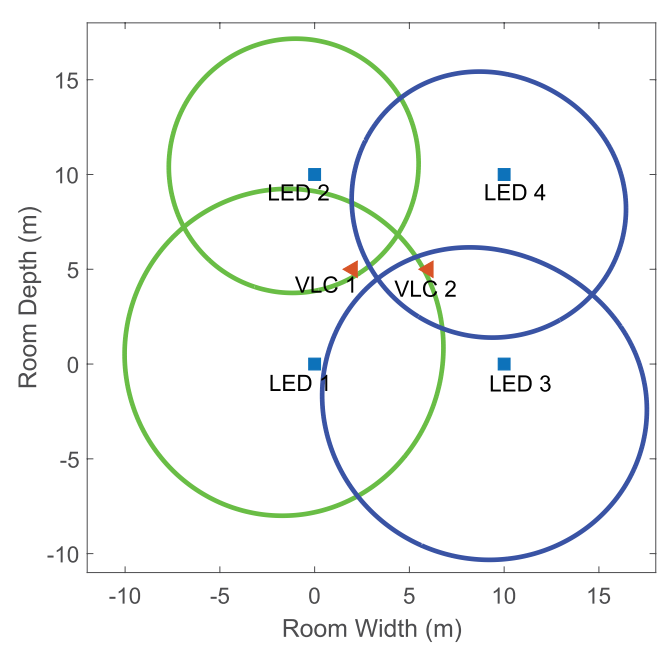

(a)

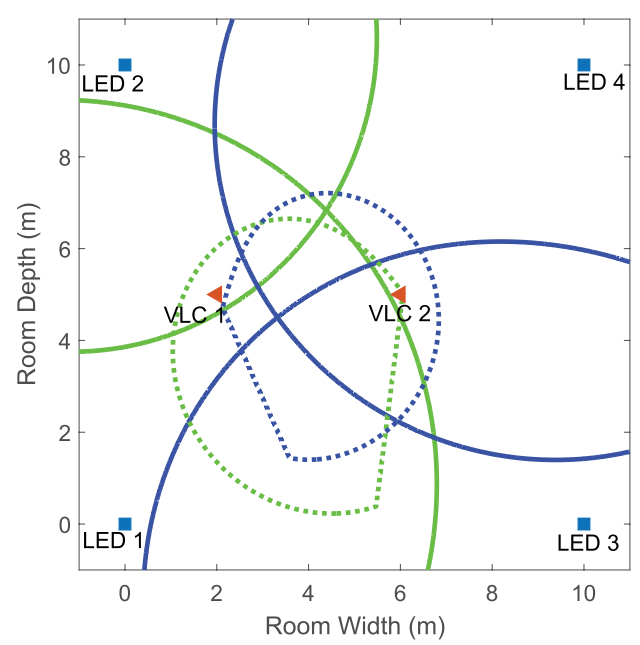

(b)

Fig. 5. (a) A noncooperative VLP system with four LED transmitters on ceiling and two VLC units, where VLC-1 gathers signals from LED-1 and LED-2, and VLC-2 from LED-3 and LED-4. The noncooperative Lambertian sets for VLC-1 and VLC-2 are represented by green and blue regions, respectively. (b) Cooperative version of the VLP system in (a), where the VLC units cooperate for improved localization performance. Green and blue dotted lines represent, respectively, the cooperative Lambertian sets corresponding to VLC-1 and VLC-2. Incorporating cooperative Lambertian sets into the localization geometry narrows the region of intersection of Lambertian sets, thereby yielding more accurate location estimates [68].

units [142]. In an asynchronous scenario, each VLC unit can sequentially update its position via the iterative step in Algorithm 1 and broadcast the resulting position information to neighboring (connected) VLC units. For the synchronous variant of Algorithm 1, VLC units can update their locations in parallel and share the updated locations with their neighbors.

To illustrate the performance of the proposed cooperative localization algorithm, we consider a VLP scenario in a room of size $10 \times 10 \times 5 \mathrm{~m}^{3}$ consisting of $L_{0}=4$ LED transmitters on the ceiling and $N_{V}=2$ VLC units, whose locations are given by $\mathbf{y}_{1}=\left[\begin{array}{lll}1 & 1 & 5\end{array}\right]^{T} \mathrm{~m}, \mathbf{y}_{2}=\left[\begin{array}{lll}1 & 9 & 5\end{array}\right]^{T} \mathrm{~m}, \mathbf{y}_{3}=\left[\begin{array}{lll}9 & 1 & 5\end{array}\right]^{T} \mathrm{~m}$, $\mathbf{y}_{4}=\left[\begin{array}{lll}9 & 9 & 5\end{array}\right]^{T} \mathrm{~m}, \mathbf{x}_{1}=\left[\begin{array}{lll}2 & 5 & 1\end{array}\right]^{T} \mathrm{~m}$, and $\mathbf{x}_{2}=\left[\begin{array}{lll}6 & 6 & 1.5\end{array}\right]^{T} \mathrm{~m}$. The LEDs on the ceiling are pointing downwards, i.e.,

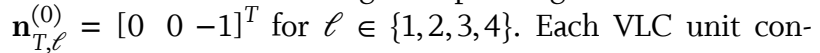
tains two PDs and one LED, whose offsets with respect to the center of the VLC unit are set to $a_{i, 1}=\left[\begin{array}{lll}0 & -0.1 & 0\end{array}\right]^{T} \mathrm{~m}$,

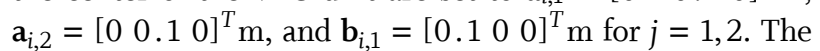
orientation vectors of the PDs and the LEDs on the VLC units are given as follows: $\mathbf{n}_{R, 1}^{(1)}=\left[\begin{array}{llll}0.3 & -0.1 & 1\end{array}\right]^{T}, \mathbf{n}_{R, 1}^{(2)}=\left[\begin{array}{llll}0.2 & 0.4 & 1\end{array}\right]^{T}$, $\mathbf{n}_{R, 2}^{(1)}=\left[\begin{array}{llll}0.8 & 0.6 & 0.1\end{array}\right]^{T}, \mathbf{n}_{R, 2}^{(2)}=\left[\begin{array}{llll}-0.7 & 0.2 & 0.1\end{array}\right]^{T}, \mathbf{n}_{T, 1}^{(1)}=\left[\begin{array}{ll}0.9 & 0\end{array}\right.$ $\left.\begin{array}{lll}0.4 & 0.1\end{array}\right]^{T}$, and $\mathbf{n}_{T, 1}^{(2)}=\left[\begin{array}{llll}-0.8 & 0.1 & 0.1\end{array}\right]^{T}$. In addition, the connectivity sets are determined as $S_{1}^{(i, j)}=\emptyset$ and $S_{2}^{(i, j)}=\{1\}$ for $i, j \in\{1,2\}, i \neq j$ for the cooperative measurements and $S_{1}^{(0,1)}=\{1,2,3\}, S_{1}^{(0,2)}=\{2,3,4\}$, and $S_{2}^{(0, j)}=\emptyset$ for $j \in\{1,2\}$ for the noncooperative measurements. Furthermore, the area of each PD is taken as $1 \mathrm{~cm}^{2}$ and the Lambertian order of all the LEDs is set to $m=1$. The noise component $\eta_{\ell, k}^{(i, j)}$ in (62) is assumed to be a zero-mean Gaussian random variable with a variance of $\sigma_{j, k}^{2}$, which is calculated using [143, Table I and eq. (6)]. The simulation results are averaged over 500 different noise realizations. In the simulations, a 2-D localization scenario is considered, i.e., the VLC units have known heights. The initial $x-y$ location of each VLC unit in Algorithm 1 is selected as the same as that of the closest LED connected to that VLC unit.

Fig. 6 shows the average localization error of the VLC units versus the transmit power of the LEDs on the ceiling achieved by Algorithm 1 and the ML estimator in (68), together with the CRLB in [68, eq. (11)], in both the noncooperative and cooperative scenarios. It is observed that cooperation among VLC units can provide significant

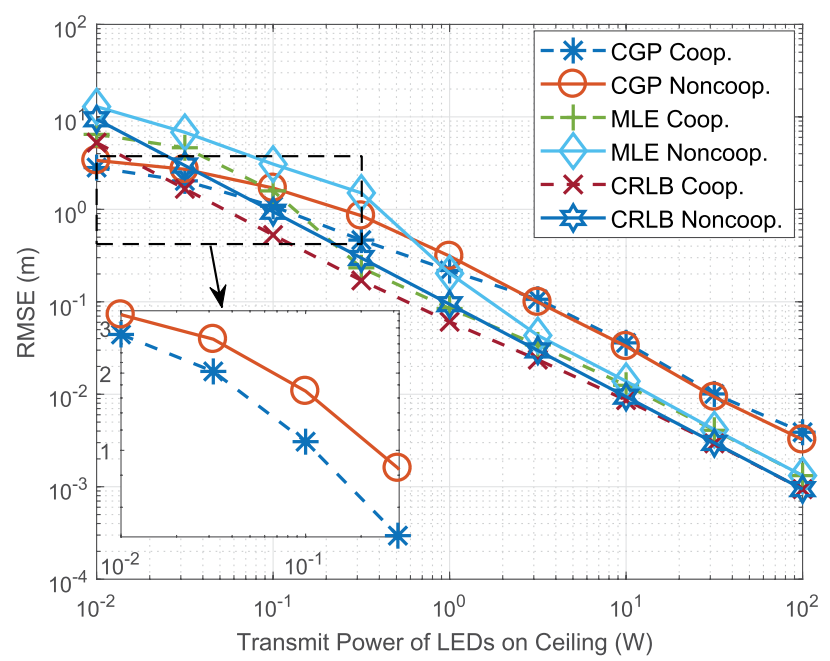

Fig. 6. Average localization error of VLC units with respect to the transmit power of LEDs on ceiling for Algorithm 1 (CGP) along with the ML estimator and the CRLB. Cooperation among VLC units provides nonnegligible performance benefits at low-to-medium LED powers. 
localization performance gains (62- and 39-cm improvements for 100- and 316-mW optical powers, respectively, via the CGP algorithm). In addition, the proposed algorithm converges to true VLC unit locations as the SNR, i.e., the transmit power of the LEDs, increases. As observed from the results in the high SNR regime, the decentralized approach (Algorithm 1) and the centralized approach [the ML estimator in (68)] can attain similar localization performance, while the former has a much lower computational complexity than the latter.

\section{OPTIMAL POWER ALLOCATION FOR LEDS UNDER ILLUMINATION CONSTRAINTS}

In VLP systems, a typical approach is to set LED transmission powers to the same constant level (e.g., [44], [45], [55], [102], and [144]), which can be adjusted according to tradeoffs among several parameters such as power consumption, localization performance, illumination concerns, and LED lifetime. However, depending on positions and orientations of LED transmitters and VLC receivers, localization accuracy can be improved by optimizing transmit powers of LEDs under certain practical considerations [69]. ${ }^{16}$ In this section, we formulate the problem of optimal power allocation for LEDs in VLP systems in the presence of some practical constraints related to power consumption and illumination requirements. In addition, based on illustrative examples, we demonstrate the improvements in localization performance that can be achieved via the optimal power allocation approach over uniform power allocation.

\section{A. Optimization Variables}

Let the transmit signal $s_{i}(t)$ for the ith LED transmitter be expressed as

$$
s_{i}(t)=\sqrt{P_{i}} \quad \tilde{s}_{i}(t)
$$

for $i=1, \ldots, N_{\mathrm{L}}$, where $N_{\mathrm{L}}$ is the number of LED transmitters, $P_{i}$ is a parameter that determines the transmit power, and $\tilde{s}_{i}(t)$ is a fixed base signal that satisfies $\int_{0}^{T_{s, i}}\left(\tilde{s}_{i}(t)\right)^{2} d t /$ $T_{s, i}=1$, with $T_{s, i}$ denoting the period of $s_{i}(t)$. Then, the optical power of $s_{i}(t)$ can be calculated as [47]

$$
E_{i}^{\text {opt }}=\frac{\int_{0}^{T_{s, i}} s_{i}(t) d t}{T_{s, i}}=\sqrt{P_{i}} \quad \tilde{E}_{i}^{\text {opt }}
$$

where

$$
\tilde{E}_{i}^{\text {opt }} \triangleq \frac{\int_{0}^{T_{s, i}} \tilde{s}_{i}(t) d t}{T_{s, i}}
$$

is a fixed quantity representing the optical power of $\tilde{s}_{i}(t)$. The electrical power of the ith LED is proportional to $P_{i}$ [93], i.e.,

$$
E_{i}^{\text {elec }} \propto \frac{\int_{0}^{T_{s, i}}\left(s_{i}(t)\right)^{2} d t}{T_{s, i}}=P_{i} .
$$

\footnotetext{
${ }^{16}$ Power allocation has been investigated for RF-based wireless localization networks in [145]-[150].
}

The goal of this section is to optimize the electrical powers of the LEDs by adjusting $\left\{P_{i}\right\}_{i=1}^{N_{\mathrm{L}}}$ to achieve improved localization accuracy.

\section{B. VLP System Constraints}

The aim of power allocation in VLP systems is to optimize the power vector $\mathbf{p} \triangleq\left[P_{1} \ldots P_{N_{\mathrm{L}}}\right]^{T} \in \mathrm{R}^{N_{\mathrm{L}}}$ subject to practical constraints so that the localization performance is maximized. In an LED power optimization scheme, the following constraints can be considered.

1) Individual Power Constraints: In order to provide efficient electrical-to-optical conversion, the LED output power must be proportional to the input drive current, which is possible if the LED transmission power operates in the linear regime [29]-[32]. In addition, high drive currents may have adverse effects on the LED lifetime by inducing self-heating [143]. Therefore, the minimum and peak power constraints for LEDs must be incorporated, resulting in the following constraint set:

$$
\mathcal{P}_{1} \triangleq\left\{\mathbf{p} \in \mathbb{R}^{N_{\mathrm{L}}}: \mathbf{p}_{\mathrm{lb}} \preccurlyeq \mathbf{p} \preccurlyeq \mathbf{p}_{\mathrm{ub}}\right\}
$$

where $\mathbf{p}_{\mathrm{lb}} \in \mathbb{R}^{N_{\mathrm{L}}}$ and $\mathbf{p}_{\mathrm{ub}} \in \mathbb{R}^{N_{\mathrm{L}}}$ represent, respectively, the lower and upper bounds on the power vector $\mathbf{p}$.

2) Total Power Constraint: As VLP systems must operate under a specific power budget, there is a certain upper limit $P_{\mathrm{T}}$ for the total electrical power of LEDs [32], [93], [151], [152]. Also, the necessity for preventing damage to human eyes leads to a limitation on the total power consumption [32]. Hence, the resulting constraint set can be defined as follows:

$$
\mathcal{P}_{2} \triangleq\left\{\mathbf{p} \in \mathbb{R}^{N_{\mathrm{L}}}: \mathbf{1}^{T} \mathbf{p} \leq P_{\mathrm{T}}\right\}
$$

where 1 denotes the all-ones vector.

3) Individual Illumination Constraints: In addition to power constraints, VLP system designers must also take into account illumination constraints to keep the brightness level at specified locations in the room above a certain threshold for proper indoor lighting [9], [22], [32], [153]. The illuminance $\left(\mathrm{lm} / \mathrm{m}^{2}, \mathrm{~lx}\right)$, defined as the luminous flux $(\mathrm{lm})$ per unit area [154], can be used as a measure of brightness. Then, utilizing [22, eq. (3)], [154, eq. (16.3)] and (86), the horizontal illuminance at location $\mathbf{x}$ due to the ith LED is obtained as

$$
\mathcal{I}_{\text {ind }}^{i}\left(\mathrm{x}, P_{i}\right)=\sqrt{P_{i}} \phi_{i}(\mathbf{x})
$$

where

$$
\phi_{i}(\mathbf{x}) \triangleq \frac{\left(m_{i}+1\right) \kappa_{i} \tilde{E}_{i}^{\mathrm{opt}}}{2 \pi} \frac{\left[\left(\mathbf{x}-\boldsymbol{l}_{\mathrm{t}}^{i}\right)^{T} \boldsymbol{n}_{\mathrm{t}}^{i}\right]^{m_{i}}\left(l_{\mathrm{t}, 3}^{i}-x_{3}\right)}{\left\|\mathbf{x}-\boldsymbol{l}_{\mathrm{t}}^{i}\right\|^{m_{i}+3}}
$$

with $\tilde{E}_{i}^{\text {opt }}$ being given by (87) and $\kappa_{i}$ denoting the luminous efficacy $(\mathrm{lm} / \mathrm{W})$ of the ith LED, defined as the optical power 
to luminous flux conversion efficiency [154]. Based on (91), the total illuminance at location $\mathbf{x}$ generated by all the LEDs can be calculated as [155]

$$
\mathcal{I}_{\text {ind }}(\mathbf{x}, \mathbf{p})=\sum_{i=1}^{N_{\mathrm{L}}} \mathcal{I}_{\text {ind }}^{i}\left(\mathbf{x}, P_{i}\right)=\sum_{i=1}^{N_{\mathrm{L}}} \sqrt{P_{i}} \phi_{i}(\mathbf{x}) .
$$

Then, the set that specifies the individual illumination constraints can be constructed as

$$
\mathcal{P}_{3} \triangleq\left\{\mathbf{p} \in \mathbb{R}^{N_{\mathrm{L}}}: \mathcal{I}_{\text {ind }}\left(\mathbf{x}_{\ell}, \mathbf{p}\right) \geq \widetilde{\mathcal{I}}_{\ell}, \ell=1, \ldots, L\right\}
$$

where $L$ is the number of locations at which the illuminance constraint is to be satisfied, and $\widetilde{\mathcal{I}}_{\ell}$ is the illuminance constraint defined for location $\mathbf{x}_{\ell}$.

4) Average Illumination Constraint: Another constraint related to illumination is the average illuminance over the room, which needs to be maintained at a certain level for satisfying average brightness requirements. The average illuminance over a horizontal region $\mathcal{A}$ in the room can be calculated as

$$
\mathcal{I}_{\text {avg }}(\mathbf{p})=\sum_{i=1}^{N_{\mathrm{L}}} \sqrt{P_{i}} \frac{\int_{\mathcal{A}} \phi_{\mathrm{i}}(\mathbf{x}) d \mathbf{x}}{|\mathcal{A}|}
$$

where $|\mathcal{A}|$ is the volume of $\mathcal{A}$. Then, the related constraint set is given by

$$
\mathcal{P}_{4} \triangleq\left\{\mathbf{p} \in \mathcal{R}^{N_{\mathrm{L}}}: \mathcal{I}_{\text {avg }}(\mathbf{p}) \geq \widetilde{\mathcal{I}}_{\text {avg }}\right\}
$$

where $\tilde{\mathcal{I}}_{\text {avg }}$ denotes the average illuminance constraint.

\section{Optimal Power Allocation for LEDs}

The accuracy of localization in VLP systems can be quantified by the CRLB on the variance of an unbiased estimate $\hat{l}_{\mathrm{r}}$ for the position of the VLC receiver $\boldsymbol{l}_{\mathrm{r}}$, which can be expressed as [69]

$$
\mathbb{E}\left\{\left\|\hat{\boldsymbol{l}}_{\mathrm{r}}-\boldsymbol{l}_{\mathrm{r}}\right\|^{2}\right\} \geq \operatorname{trace}\left\{\mathrm{J}^{-1}(\mathbf{p})\right\}
$$

where $\mathbf{J}(\mathbf{p})$ is the FIM given by

$$
\mathbf{J}(\mathbf{p})=\left(\mathbf{I}_{3} \otimes \mathbf{p}\right)^{T} \Gamma .
$$

In (98), $\otimes$ denotes the Kronecker product, $\mathbf{I}_{3}$ is a $3 \times 3$ identity matrix, and $\Gamma \in \mathbb{R}^{3 \mathrm{~N}_{\mathrm{L}} \times 3}$ is a known matrix that is independent of $\mathbf{p}$ and depends on VLP system parameters [69]. ${ }^{17}$

For LED power optimization, the CRLB is chosen as the optimization metric for evaluating the localization performance since it can be achieved asymptotically by the ML location estimator as the SNR and/or effective bandwidth increases [97]. Hence, considering the system constraints in Section IV-B, the optimal power allocation problem for LED transmitters is formulated as

$$
\begin{array}{ll}
\underset{\mathbf{p}}{\operatorname{minimize}} & \operatorname{trace}\left\{\mathbf{J}^{-1}(\mathbf{p})\right\} \\
\text { subject to } & \mathbf{p} \in \mathcal{P}
\end{array}
$$

${ }^{17}$ The CRLB expression in (97) and (98) is also valid for scenarios in which the VLC receiver is connected to a subset of all the LEDs in the VLP system. where $\mathcal{P} \triangleq \cap_{i=1}^{4} \mathcal{P}_{i}$. The aim of (99) is to derive the optimal LED power distribution that minimizes the CRLB for the localization of a VLC receiver under power and illumination constraints. As the optimization problem in (99) is convex due to the convexity of the objective function in (99a) and of the constraint sets in (89), (90), (94), and (96) [69], the optimal solution can be obtained efficiently by employing standard tools of convex optimization, e.g., interior-point methods [156].

A related problem of interest is to minimize the total power consumption subject to a prescribed level of localization accuracy, which can formally be stated as

$$
\begin{array}{ll}
\underset{\mathbf{p}}{\operatorname{minimize}} & \mathbf{1}^{T} \mathbf{p} \\
\text { subject to } & \operatorname{trace}\left\{\mathbf{J}^{-1}(\mathbf{p})\right\} \leq \varepsilon \\
& \mathbf{p} \in \mathcal{P}_{S}
\end{array}
$$

where $\mathcal{P}_{S} \triangleq \mathcal{P}_{1} \cap \mathcal{P}_{3} \cap \mathcal{P}_{4}$ and $\varepsilon$ represents the maximum tolerable CRLB level for the localization of the VLC receiver. Similar to (99), the problem in (100) is convex.

In the problem formulations (99) and (100), optimizing the power vector $p$ requires the knowledge of $\Gamma$ in (98), which involves parameters such as the location and orientation of the VLC receiver [69]. In most practical VLP scenarios, those parameters cannot perfectly be estimated (e.g., due to localization and tracking errors, and gyroscope measurement errors), which may lead to unsatisfactory power allocation results. Therefore, we also need to consider robust power allocation formulations in the presence of uncertainties in VLP system parameters. To that aim, let $\hat{l}_{\mathrm{r}}$ be the estimated location of the VLC receiver, given as

$$
\hat{l}_{\mathrm{r}}=l_{\mathrm{r}}+\mathrm{e}_{l_{\mathrm{r}}}
$$

where $\boldsymbol{l}_{\mathrm{r}}$ is the true location and $\mathbf{e}_{\boldsymbol{l}_{\mathrm{r}}}$ is the error vector. Assuming a spherical uncertainty set for the location errors [157], [158], i.e.,

$$
\mathbf{e}_{\mathbf{l}_{\mathrm{r}}} \in \mathcal{E}_{\mathbf{l}_{\mathrm{r}}} \triangleq\left\{\mathbf{e} \in \mathbb{R}^{3}:\|\mathrm{e}\| \leq \delta_{\boldsymbol{l}_{\mathrm{r}}}\right\}
$$

where $\delta_{I_{\mathrm{r}}}$ denotes the maximum error in the location of the VLC receiver, the robust counterpart of the power allocation problem in (99) under location uncertainties can be formulated as

$$
\begin{aligned}
& \underset{\mathbf{p}}{\operatorname{minimize}} \max _{\mathbf{e}_{l_{r}} \in \mathcal{E}_{\boldsymbol{I}_{\mathrm{r}}}} \operatorname{trace}\left\{\left(\left(\mathbf{I}_{3} \otimes \mathbf{p}\right)^{T} \Gamma\left(\hat{\boldsymbol{l}}_{\mathrm{r}}-\mathbf{e}_{\mathbf{l}_{\mathrm{r}}}\right)\right)^{-1}\right\} \\
& \text { subject to } \mathbf{p} \in \mathcal{P}
\end{aligned}
$$

with $\Gamma\left(\hat{\boldsymbol{l}}_{\mathrm{r}}-\mathbf{e}_{\boldsymbol{l}_{\mathrm{r}}}\right)$ corresponding to the matrix $\Gamma$ in (98) evaluated at $\boldsymbol{l}_{\mathrm{r}}=\hat{l}_{\mathrm{r}}-\mathbf{e}_{\boldsymbol{l}_{\mathrm{r}}}$. The goal of robustness in (103) is to minimize the worst case CRLB over the uncertainty region $\mathcal{E}_{\boldsymbol{l}_{\mathrm{r}}}$ in (102) using the estimated location $\hat{\boldsymbol{l}}_{\mathrm{r}}$. Similar formulations to that of (103) can be developed for handling the uncertainty in other parameters (e.g., orientation of the VLC 
receiver), as well [69]. By exploiting the characteristics of the objective function, efficient iterative algorithms can be designed to solve (103) (see [69, Sec. V] for details).

It is worth noting that as in both of the optimization problems in (99) and (100), the CRLBs in VLP systems are different from those in RF-based systems since the system model in VLP scenarios has significant differences compared to the one in RF-based systems, as discussed in Section II-A1. In addition to the CRLB definitions, the constraint sets in (99) and (100) are also different as they include individual and average illumination constraints, which are not considered in the design of RF-based localization systems.

\section{Simulation Examples}

To illustrate the benefits of optimal allocation of LED powers in VLP systems, we consider a simple localization scenario as depicted in Fig. 7, where there exist four LED transmitters $\left(N_{\mathrm{L}}=4\right)$ on the ceiling of a room of size $5 \times 5 \times 2.5 \mathrm{~m}^{3}$ and a VLC receiver trying to localize itself based on signals transmitted by the LEDs. The locations of the LED transmitters and the VLC receiver are given by $\boldsymbol{l}_{\mathrm{t}}^{1}=\left[\begin{array}{lll}1 & 1 & 2.5\end{array}\right]^{T} \mathrm{~m}, \boldsymbol{l}_{\mathrm{t}}^{2}=\left[\begin{array}{lll}1 & 4 & 2.5\end{array}\right]^{T} \mathrm{~m}, \boldsymbol{l}_{\mathrm{t}}^{3}=\left[\begin{array}{lll}4 & 1 & 2.5\end{array}\right]^{\mathrm{T}} \mathrm{m}$, $\boldsymbol{l}_{\mathrm{t}}^{4}=\left[\begin{array}{lll}4 & 4 & 2.5\end{array}\right]^{T} \mathrm{~m}$, and $\boldsymbol{l}_{\mathrm{r}}=\left[\begin{array}{llll}2 & 0 & .5 & 1\end{array}\right]^{\mathrm{T}} \mathrm{m}$. The LED transmitters have perpendicular orientations, i.e., $\boldsymbol{n}_{\mathrm{t}}^{i}=\left[\begin{array}{lll}0 & 0 & -1\end{array}\right]^{T}$ for $i=1,$. . ., $N_{\mathrm{L}}$, while the orientation of the VLC receiver is given by $\boldsymbol{n}_{\mathrm{r}}=[-0.2241-0.12940 .9659]^{T}$, which corresponds to an elevation angle of $15^{\circ}$ and an azimuth angle of $210^{\circ}$. Regarding the illumination requirements, we determine four locations for the individual illumination constraints, which are specified by $\mathbf{x}_{1}=\left[\begin{array}{llll}1.5 & 1.5 & 1.5\end{array}\right]^{T} \mathrm{~m}$, $\mathbf{x}_{2}=\left[\begin{array}{llll}1.5 & 3.5 & 1.5\end{array}\right]^{T} \mathrm{~m}, \quad \mathbf{x}_{3}=\left[\begin{array}{llll}3.5 & 1.5 & 1.5\end{array}\right]^{T} \mathrm{~m}$, and $\mathbf{x}_{4}=\left[\begin{array}{llll}3.5 & 3.5 & 1.5\end{array}\right]^{T} \mathrm{~m}$.

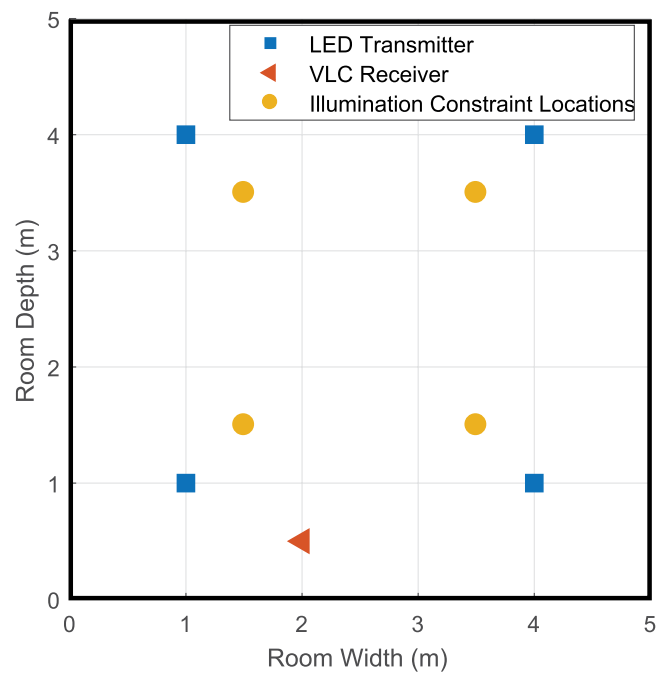

Fig. 7. VLP scenario with four LED transmitters and a VLC receiver, shown in 2-D.
For the LED transmitters, the Lambertian order is set to $m_{i}=3$ and the luminous efficacy is $\kappa_{i}=60 \mathrm{~lm} / \mathrm{W}$ for $i=$ $1, \ldots, N_{\mathrm{L}}$ [153]. Also, the area of the PD at the VLC receiver is taken as $A_{R}=0.64 \mathrm{~cm}^{2}$, the responsivity of the PD is set to $R_{p}=0.4 \mathrm{~mA} / \mathrm{mW}$, and the spectral density level of the noise is $\sigma^{2}=8.5641 \times 10^{-23} \mathrm{~W} / \mathrm{Hz}$ [47]. The base transmit signal $\tilde{s}_{i}(t)$ in (85) is modeled as [47]

$$
\tilde{s}_{i}(t)=\frac{2}{3}\left(1-\cos \left(2 \pi t / T_{s, i}\right)\right)\left(1+\cos \left(2 \pi f_{c, i} t\right)\right)
$$

for $i=1, \ldots, N_{\mathrm{L}}$ and $t \in\left[0, T_{s, i}\right]$, where $f_{c, i}$ is the center frequency and $T_{s, i}$ denotes the observation interval. ${ }^{18}$ For the signal model in (104), $\tilde{E}_{i}^{\text {opt }}$ in (87) is obtained as $\tilde{E}_{i}^{\text {opt }}=2 / 3$. For the simulations, an asynchronous VLP system is considered with $f_{c, i}=10 i \mathrm{MHz}$ and $T_{s, i}=1 \mu \mathrm{s}$ for $i=1, \ldots$, $N_{\mathrm{L}}$. In addition, the lower and upper bounds on the LED optical powers are set as $1 \mathrm{~W}$ and $10 \mathrm{~W}$, which, based on (86) and (87), correspond to power limits of $p_{\mathrm{lb}, i}=2.25$ and $p_{\mathrm{ub}, i}=225$ for $i=1, \ldots, N_{\mathrm{L}}$ for the constraint set $\mathcal{P}_{1}$ in (89).

In Fig. 8, the square root of the CRLBs achieved by the optimal power allocation strategy of (99) and the uniform strategy are plotted with respect to $P_{\mathrm{T}} / N_{\mathrm{L}}$, which determines the average electrical power limit. The illumination constraints are set as $\tilde{\mathcal{I}}_{\text {avg }}=\tilde{\mathcal{I}}_{\ell}=20 \mathrm{~lx}$ for $\ell=1,2,3,4$. It is deduced from Fig. 8 that the optimal power allocation approach can provide significant improvements in localization performance over the uniform strategy. For low power budgets, the problem in (99) becomes infeasible due to the illumination constraints. On the other hand, for sufficiently high $P_{\mathrm{T}}$, the optimal and uniform strategies become equivalent as they both assign the peak powers (i.e., $\mathbf{p}_{\mathrm{ub}}$ ) to the LEDs. In addition, Fig. 9 shows the CRLBs of the optimal and uniform strategies corresponding to (99) for a constant

${ }^{18}$ The constant factor $2 / 3$ is included for satisfying $\int_{0}^{T_{s, i}}\left(\tilde{s}_{i}(t)\right)^{2} d t /$ $T_{s, i}=1$, as mentioned in Section IV-A.

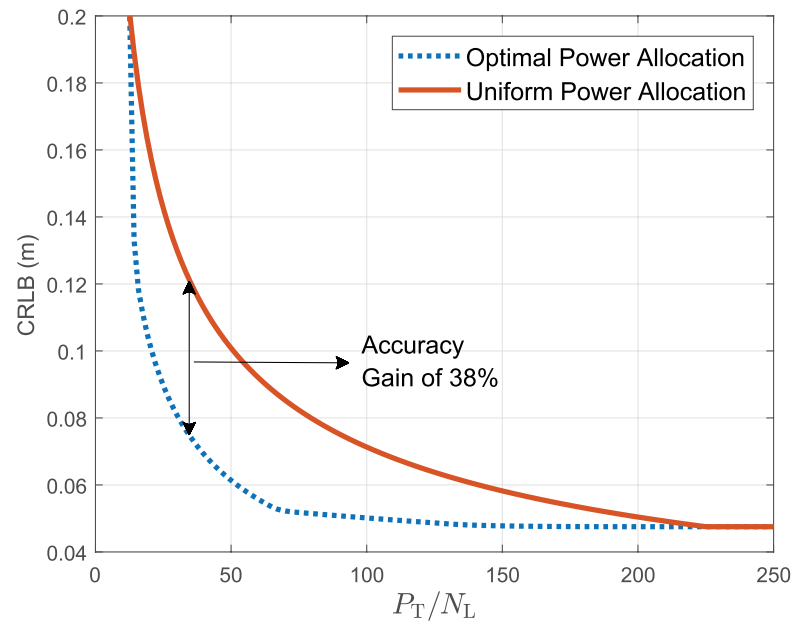

Fig. 8. CRLB of (99a) versus $P_{T} / N_{L}$ for uniform and optimal power allocation strategies, where the average and individual illumination constraints are taken as $\tilde{\mathcal{I}}_{\text {avg }}=\tilde{\mathcal{I}}_{\ell}=20 \mathrm{~lx}$ for $\ell=1,2,3,4$. 


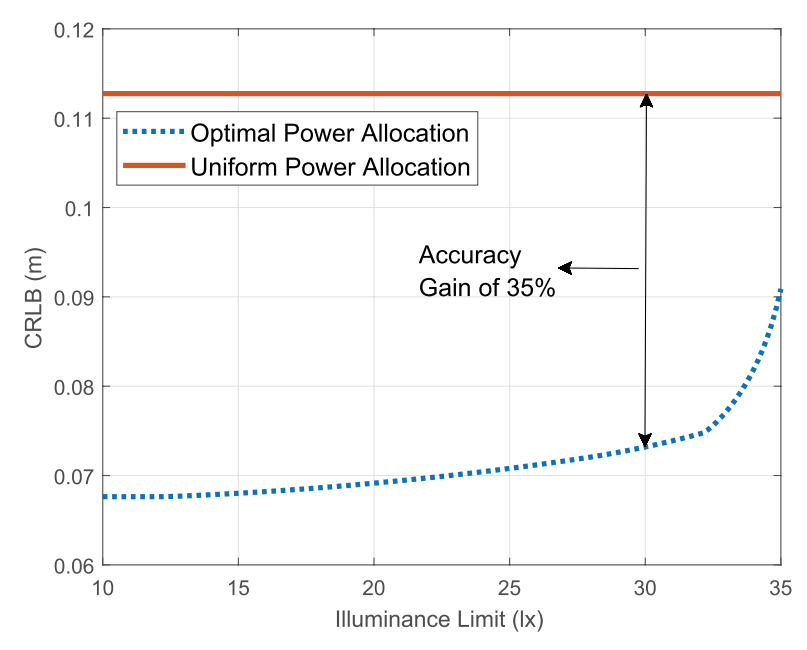

Fig. 9. CRLB of (99a) versus the illuminance limit for uniform and optimal power allocation strategies, where the average power constraint is set to $P_{T} / N_{L}=40$.

average power limit $P_{\mathrm{T}} / N_{\mathrm{L}}=40$ as the illuminance limits $\tilde{\mathcal{I}}_{\text {avg }}$ and $\mathcal{I}_{\ell}$ vary. It is observed that the accuracy improvement via power optimization gets higher as the illumination constraints become less stringent, as expected.

In the second example, we investigate the optimal value of (100a), which determines the total electrical power consumption, against the desired CRLB level $\sqrt{\varepsilon}$ in (100b) under various illumination requirements. The results are shown in Fig. 10, where the $y$-axis is calculated as $P_{\mathrm{T}}^{\star} / N_{\mathrm{L}}$ with $P_{\mathrm{T}}^{\star}$ denoting the optimal value of the objective function in (100a). It is observed that substantial power saving gains can be obtained via the optimal approach as compared to the conventional uniform approach. Also, the optimal strategy coincides with the uniform strategy for sufficiently high values of the desired CRLB level due to the illumination constraints. In summary, given a desired level of localization

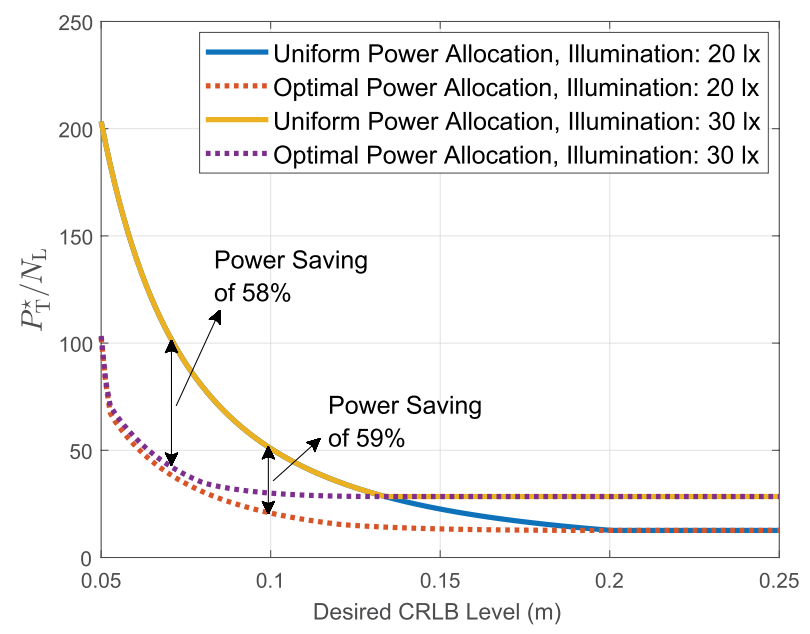

Fig. 10. Optimal value of (100a) divided by $N_{L}\left(P_{T} / N_{L}\right)$ versus the desired CRLB level $\sqrt{\varepsilon}$ in (100b) for uniform and optimal power allocation strategies under various illumination constraints. accuracy in a VLP system, the proposed optimal approach can provide a much more power efficient solution than the uniform approach.

\section{CONCLUSION AND FUTURE RESEARCH DIRECTIONS}

As a key enabler for low-cost and high-accuracy indoor wireless localization services, the VLP technology constitutes a vital ingredient of next-generation locationaware applications. Therefore, it is imperative, for both researchers in the academia and practical system designers in the industry, to acquire a meticulous understanding of the fundamental trends in position estimation via visible light signals and their impacts on the performance of VLP systems under various operation environments. In this paper, we have considered the problem of localization in visible light systems and provided a survey of the state-of-the-art techniques by taking into account the two main research strands. Starting with a received signal model for VLC signals, we have presented the direct positioning approach utilized in synchronous, quasi-synchronous, and asynchronous VLP systems, and provided the performance benchmarks that can be used as guidelines for system design engineers. Then, we have considered the two-step approach to localization, which consists of parameter estimation/extraction and position estimation steps. Regarding parameter estimation, we have investigated the properties of the widely used first-step parameters in VLP systems, such as RSS, TOA, TDOA, and AOA, and discussed the estimation methods (e.g., the ML estimates of these parameters) and hardware-related requirements imposed by specific types of parameters (e.g., multiple PDs at the VLC receiver for the case of AOA, and synchronization for the case of TOA and TDOA). For the position estimation step, proximity-based methods, geometric methods, statistical methods, and fingerprinting methods have been reviewed, with an emphasis on statistical position estimators as they provide a mathematically rigorous framework for position estimation, which provides asymptotic performance guarantees.

In addition, we have devised a cooperative VLP system architecture that utilizes communications among VLC receiver units to improve the accuracy of localization via cooperation. A cooperative localization algorithm that is amenable to distributed implementation has been proposed to illustrate the improvements in localization performance via the use of cooperation among the VLC units. Finally, we have considered optimal LED power allocation strategies to maximize the localization accuracy of VLC receivers subject to power and illumination constraints. The problem of optimal power allocation has been shown to be formulated as a convex program, on the basis of which the optimal power vectors have been derived efficiently to showcase the 
performance benefits over the conventional uniform power allocation approach.

Although significant improvements are being made for VLP systems in the literature, there are still some issues which have not adequately been addressed and should be investigated in future work. A recent study in the literature has shown that omitting multipath reflections in VLP systems may considerably reduce the accuracy of localization in certain indoor environments [99]. For that reason, VLP systems should be designed in consideration of multipath propagation. In a similar context, the common algorithms and methods in the literature do not consider the situation when the LOS between the LED transmitter and the VLC receiver is lost; that is, when an LOS blockage occurs. Regarding this issue, the approaches for VLP systems should be adapted for the case of LOS blockage. In addition, the VLP systems should be invulnerable to various interference sources such as sunlight and other lighting systems. Moreover, most VLP systems cannot be treated separately from illumination systems, and consequently the design of such systems requires the consideration of not only localization performance but also illumination constraints. Furthermore, in scenarios with mobile entities, temporal cooperation can be utilized by taking into account the previous state information of a VLC receiver in the design of VLP algorithms in order to achieve robust localization results. Overall, in view of these challenges and remarks, fully integrated superior designs can be developed for VLP systems in the future.

\section{REFERENCES}

[1] J. Armstrong, Y. A. Sekercioglu, and A. Neild, "Visible light positioning: A roadmap for international standardization," IEEE Commun. Mag., vol. 51, no. 12, pp. 68-73, Dec. 2013.

[2] Y. Gu, A. Lo, and I. Niemegeers, "A survey of indoor positioning systems for wireless personal networks," IEEE Commun. Surveys Tuts., vol. 11, no. 1, pp. 13-32, 1st Quart., 2009.

[3] H. Burchardt, N. Serafimovski, D. Tsonev, S. Videv, and H. Haas, "VLC: Beyond pointto-point communication," IEEE Commun. Mag., vol. 52, no. 7, pp. 98-105, Jul. 2014.

[4] P. H. Pathak, X. Feng, P. Hu, and P. Mohapatra, "Visible light communication, networking, and sensing: A survey, potential and challenges," IEEE Commun. Surveys Tuts. vol. 17, no. 4, pp. 2047-2077, 4th Quart., 2015.

[5] S. Rajagopal, R. D. Roberts, and S.-K. Lim, "IEEE 802.15.7 visible light communication: Modulation schemes and dimming support," IEEE Commun. Mag., vol. 50, no. 3, pp. 72-82, Mar. 2012.

[6] D. Karunatilaka, F. Zafar, V. Kalavally, and R. Parthiban, "LED based indoor visible light communications: State of the art," IEEE Commun. Surveys Tuts., vol. 17, no. 3, pp. 1649-1678, 3rd Quart., 2015.

[7] A. Jovicic, J. Li, and T. Richardson, "Visible light communication: Opportunities, challenges and the path to market," IEEE Commun. Mag., vol. 51, no. 12, pp. 26-32, Dec. 2013.

[8] Z. Ghassemlooy, L. N. Alves, S. Zvanovec, and M.-A. Khalighi, Visible Light Communications: Theory and Applications. Boca Raton, FL, USA: CRC Press, 2017.

[9] A. Tsiatmas, C. P. M. J. Baggen, F. M. J. Willems, J.-P. M. G. Linnartz, and J. W. M. Bergmans, "An illumination perspective on visible light communications," IEEE Commun. Mag., vol. 52, no. 7, pp. 64-71, Jul. 2014.

[10] L. Yin and H. Haas, "Physical-layer security in multiuser visible light communication networks," IEEE J. Sel. Areas Commun., vol. 36, no. 1, pp. 162-174, Jan. 2018.

[11] G. Seco-Granados, J. López-Salcedo, D. Jiménez-Baños, and G. López-Risueño, "Challenges in indoor global navigation satellite systems: Unveiling its core features in signal processing," IEEE Signal Process. Mag., vol. 29, no. 2, pp. 108-131, Mar. 2012.
[12] H. Liu, H. Darabi, P. Banerjee, and J. Liu, "Survey of wireless indoor positioning techniques and systems," IEEE Trans. Syst. Man Cybern. C, Appl. Rev., vol. 37, no. 6, pp. 1067-1080, Nov. 2007.

[13] K. Pahlavan, X. Li, and J.-P. Mäkelä, "Indoor geolocation science and technology," IEEE Commun. Mag., vol. 40, no. 2, pp. 112-118, Feb. 2002.

[14] S. Li, M. Hedley, and I. B. Collings, "New efficient indoor cooperative localization algorithm with empirical ranging error model," IEEE J. Sel. Areas Commun., vol. 33, no. 7, pp. 1407-1417, Jul. 2015.

[15] A. Conti, D. Dardari, M. Guerra, L. Mucchi, and M. Z. Win, "Experimental characterization of diversity navigation," IEEE Syst. J., vol. 8, no. 1, pp. 115-124, Mar. 2014.

[16] T. Komine and M. Nakagawa, "Fundamental analysis for visible-light communication system using LED lights," IEEE Trans. Consum. Electron., vol. 50, no. 1, pp. 100-107, Feb. 2004.

[17] T.-H. Do and M. Yoo, "An in-depth survey of visible light communication based positioning systems," Sensors, vol. 16, no. 5, p. $678,2016$.

[18] B. Xie et al., "LIPS: A light intensity-based positioning system for indoor environments," ACM Trans. Sensor Netw., vol. 12, no. 4, p. 28, 2016

[19] D. Dardari, A. Conti, U. Ferner, A. Giorgetti, and M. Z. Win, "Ranging with ultrawide bandwidth signals in multipath environments," Proc. IEEE, vol. 97, no. 2 , pp. 404-426, Feb. 2009.

[20] S. Marano, W. M. Gifford, H. Wymeersch and M. Z. Win, "NLOS identification and mitigation for localization based on UWB experimental data," IEEE J. Sel. Areas Commun., vol. 28, no. 7, pp. 1026-1035, Sep. 2010.

[21] S. Bartoletti, A. Giorgetti, M. Z. Win, and A. Conti, "Blind Selection of Representative Observations for Sensor Radar Networks," IEEE Trans. Veh. Technol., vol. 64, no. 4, pp. 1388-1400, Apr. 2015.

[22] J. Grubor, S. Randel, K.-D. Langer, and J. W. Walewski, "Broadband information broadcasting using LED-based interior lighting," J. Lightw. Technol., vol. 26, no. 24, pp. 3883-3892, Dec. 15, 2008.

[23] A.-M. Cilean and M. Dimian, "Current challenges for visible light communications usage in vehicle applications: A survey," IEEE Commun. Surveys Tuts., vol. 19, no. 4, pp. 2681-2703, 4th Quart., 2017.

[24] T. H. Do and M. Yoo, "Potentialities and challenges of VLC based outdoor positioning," in Proc. Int. Conf. Inf. Netw. (ICOIN), Jan. 2015, pp. 474-477.

[25] S. Schmid, T. Richner, S. Mangold, and T. R. Gross, "EnLighting: An indoor visible light communication system based on networked light bulbs," in Proc. 13th Annu. IEEE Int. Conf. Sens. Commun. Netw. (SECON), Jun. 2016, pp. 1-9.

[26] R. Roberts, P. Gopalakrishnan, and S. Rathi, "Visible light positioning: Automotive use case," in Proc. IEEE Veh. Netw. Conf. (VNC), Dec. 2010, pp. 309-314.

[27] B. Béchadergue, L. Chassagne, and H. Guan, "Visible light phase-shift rangefinder for platooning applications," in Proc. IEEE 19th Int. Conf. Intell. Transp. Syst. (ITSC), Nov. 2016, pp. 2462-2468.

[28] K. Qiu, F. Zhang, and M. Liu, "Let the light guide us: VLC-based localization," IEEE Robot. Autom. Mag., vol. 23, no. 4, pp. 174-183, Dec. 2016.

[29] H. Elgala, R. Mesleh, and H. Haas, "An LED model for intensity-modulated optical communication systems," IEEE Photon. Technol. Lett., vol. 22, no. 11, pp. 835-837, Jun. 1, 2010.

[30] C. Gong, S. Li, Q. Gao, and Z. Xu, "Power and rate optimization for visible light communication system with lighting constraints," IEEE Trans. Signal Process., vol. 63, no. 16, pp. 4245-4256, Aug. 2015.

[31] T. D. C. Little and H. Elgala, "Adaptation of OFDM under visible light communications and illumination constraints," in Proc. 48th Asilomar Conf. Signals Syst. Comput., Nov. 2014, pp. 1739-1744.

[32] J. Gancarz, H. Elgala, and T. D. C. Little, "Impact of lighting requirements on VLC systems," IEEE Commun. Mag., vol. 51, no. 12, pp. 34-41, Dec. 2013.

[33] A. H. Sayed, A. Tarighat, and N. Khajehnouri, "Network-based wireless location: Challenges faced in developing techniques for accurate wireless location information," IEEE Signal Process. Mag., vol. 22, no. 4, pp. 24-40, Jul. 2005.

[34] M. Z. Win et al., "Network localization and navigation via cooperation," IEEE Commun. Mag., vol. 49, no. 5, pp. 56-62, May 2011. 
[35] S. Gezici et al., "Localization via ultrawideband radios: A look at positioning aspects for future sensor networks," IEEE Signal Process. Mag., vol. 22, no. 4 pp. 70-84, Jul. 2005.

[36] Y. Shen, S. Mazuelas, and M. Z. Win, "Network navigation: Theory and interpretation," IEEE J. Sel. Areas Commun., vol. 30, no. 9, pp. 1823-1834, Oct. 2012.

[37] Y. Shen and M. Z. Win, "Fundamental limits of wideband localization-Part I: A general framework," IEEE Trans. Inf. Theory, vol. 56, no. 10, pp. 4956-4980, Oct. 2010.

[38] S. Bartoletti, W. Dai, A. Conti, and M. Z. Win, "A mathematical model for wideband ranging," IEEE J. Sel. Topics Signal Process., vol. 9, no. 2, pp. 216-228, Mar. 2015.

[39] S. Mazuelas, A. Conti, J. Allen, and M. Win, "Soft range information for network localization," IEEE Trans. Signal Process., to be published.

[40] W. Xu, J. Wang, H. Shen, H. Zhang, and $\mathrm{X}$. You, "Indoor positioning for multiphotodiode device using visible-light communications," IEEE Photon. J., vol. 8, no. 1, Feb. 2016, Art. no. 7900511.

[41] T. Tanaka and S. Haruyama, "New position detection method using image sensor and visible light LEDs," in Proc. 2nd Int. Conf. Mach. Vis., Dec. 2009, pp. 150-153.

[42] M. Yoshino, S. Haruyama, and M. Nakagawa, "High-accuracy positioning system using visible LED lights and image sensor," in Proc. IEEE Radio Wireless Symp., Jan. 2008, pp. 439-442.

[43] T.-H. Do and M. Yoo, "Analysis on visible light communication using rolling shutter CMOS sensor," in Proc. Int. Conf. Inf. Commun. Technol. Converg. (ICTC), Oct. 2015, pp. 755-757.

[44] H.-S. Kim, D.-R. Kim, S.-H. Yang, Y.-H. Son and S.-K. Han, "An indoor visible light communication positioning system using a RF carrier allocation technique," J. Lightw. Technol., vol. 31, no. 1, pp. 134-144, Jan. 1 , 2013.

[45] W. Zhang, M. I. S. Chowdhury, and M. Kavehrad, "Asynchronous indoor positioning system based on visible light communications," Opt. Eng., vol. 53, no. 4, pp. 045105-1-045105-9, 2014.

[46] L. Li, P. Hu, C. Peng, G. Shen, and F. Zhao, "Epsilon: A visible light based positioning system," in Proc. 11th USENIX Symp. Netw. Syst. Design Implement. (NSDI), Seattle, WA, USA, Apr. 2014, pp. 331-343.

[47] T. Q. Wang, Y. A. Sekercioglu, A. Neild, and J. Armstrong, "Position accuracy of time-ofarrival based ranging using visible light with application in indoor localization systems," J. Lightw. Technol., vol. 31, no. 20, pp. 3302-3308, Oct. 15, 2013.

[48] M. F. Keskin and S. Gezici, "Comparative theoretical analysis of distance estimation in visible light positioning systems," J. Lightw. Technol., vol. 34, no. 3 , pp. 854-865, Feb. 1, 2016.

[49] M. F. Keskin, E. Gonendik, and S. Gezici, "Improved lower bounds for ranging in synchronous visible light positioning systems," J. Lightw. Technol., vol. 34, no. 23, pp. 5496-5504, Dec. 1, 2016.

[50] O. J. Pandey, R. Sharan, and R. M. Hegde, "Localization in wireless sensor networks using visible light in non-line of sight conditions," Wireless Pers. Commun., vol. 97, pp. 6519-6539, Dec. 2017.
[51] T.-H. Do and M. Yoo, "TDOA-based indoor positioning using visible light," Photon. Netw. Commun., vol. 27, no. 2, pp. 80-88, 2014.

[52] S.-Y. Jung, S. Hann, and C.-S. Park, "TDOAbased optical wireless indoor localization using LED ceiling lamps," IEEE Trans. Consum. Electron., vol. 57, no. 4, pp. 15921597, Nov. 2011.

[53] S. Lee and S.-Y. Jung, "Location awareness using Angle-of-arrival based circular-PDarray for visible light communication," in Proc. 18th Asia-Pacific Conf. Commun. (APCC), Oct. 2012, pp. 480-485.

[54] Y. U. Lee and S.-M. Lee, "Random distributed angle-of-arrival parameter estimation technique for visible light positioning," in Proc. 38th Int. Conf. Telecommun. Signal Process. (TSP), 2015, pp. 467-471.

[55] A. Şahin, Y. S. Eroglu, I. Güvenç, N. Pala and M. Yüksel, "Hybrid 3-D localization for visible light communication systems," J. Lightw. Technol., vol. 33, no. 22, pp. 4589-4599, Nov. 15, 2015

[56] H. Steendam, "A 3-D positioning algorithm for AOA-based VLP with an aperture-based receiver," IEEE J. Sel. Areas Commun., vol. 36, no. 1, pp. 23-33, Jan. 2018

[57] Y. U. Lee and M. Kavehrad, “Two hybrid positioning system design techniques with lighting LEDs and ad-hoc wireless network," IEEE Trans. Consum. Electron., vol. 58, no. 4, pp. 1176-1184, Nov. 2012.

[58] M. Nakajima and S. Haruyama, "New indoor navigation system for visually impaired people using visible light communication," EURASIP J. Wireless Commun. Netw., vol. 2013, no. 1, p. 37, Feb. 2013.

[59] M. Aminikashani, W. Gu, and M. Kavehrad, "Indoor positioning with OFDM visible light communications," in Proc. 13th IEEE Annu. Consum. Commun. Netw. Conf. (CCNC), Jan. 2016, pp. 505-510.

[60] Y. S. Eroglu, I. Güvenç, N. Pala, and M. Yüksel, "AOA-based localization and tracking in multi-element VLC systems," in Proc. IEEE 16th Annu. Wireless Microw. Technol. Conf. (WAMICON), Apr. 2015, pp. 1-5.

[61] S. Gezici, "A survey on wireless position estimation," Wireless Personal Commun. vol. 44, no. 3, pp. 263-282, Feb. 2008.

[62] H. V. Poor, An Introduction to Signal Detection and Estimation. New York, NY, USA: Springer-Verlag, 1994.

[63] M. F. Keskin, S. Gezici, and O. Arikan, "Direct and two-step positioning in visible light systems," IEEE Trans. Commun., vol. 66, no. 1, pp. 239-254, Jan. 2018.

[64] J. Lim, "Ubiquitous 3D positioning systems by LED-based visible light communications," IEEE Wireless Commun., vol. 22, no. 2, pp. 80-85, Apr. 2015.

[65] S. Feng, X. Li, R. Zhang, M. Jiang, and L. Hanzo, "Hybrid positioning aided amorphous-cell assisted user-centric visible light downlink techniques," IEEE Access, vol. 4, pp. 2705-2713, 2016.

[66] J. Vongkulbhisal, B. Chantaramolee, Y. Zhao, and W. S. Mohammed, "A fingerprinting based indoor localization system using intensity modulation of light emitting diodes," Microw. Opt. Technol. Lett., vol. 54, no. 5, pp. 1218-1227, 2012

[67] O. Erdem, M. F. Keskin, and S. Gezici, "Effects of cooperation on visible light positioning," in Proc. IEEE Int. Black Sea Conf. Commun. Netw. (BlackSeaCom), Jun. 2017, pp. 1-5.
[68] M. F. Keskin, O. Erdem, and S. Gezici (2018). "Cooperative localization in visible light networks: Theoretical limits and distributed algorithms." [Online]. Available: https://arxiv.org/abs/1804.00576

[69] M. F. Keskin, A. D. Sezer, and S. Gezici (2018). "Optimal and robust power allocation for visible light positioning systems under illumination constraints." [Online]. Available: https://arxiv.org/ abs/1802.04505

[70] H. Wymeersch, J. Lien, and M. Z. Win, "Cooperative localization in wireless networks," Proc. IEEE, vol. 97, no. 2, pp. 427-450, Feb. 2009.

[71] Y. Shen, H. Wymeersch, and M. Z. Win, "Fundamental limits of wideband localization-Part II: Cooperative networks," IEEE Trans. Inf. Theory, vol. 56, no. 10, pp. 4981-5000, Oct. 2010.

[72] F. Meyer, H. Wymeersch, M. Fröhle, and F. Hlawatsch, "Distributed estimation with information-seeking control in agent networks," IEEE J. Sel. Areas Commun., vol. 33, no. 11, pp. 2439-2456, Nov. 2015.

[73] Y.-S. Kuo, P. Pannuto, K.-J. Hsiao, and P. Dutta, "Luxapose: Indoor positioning with mobile phones and visible light," in Proc. 20th Annu. Int. Conf. Mobile Comput. Netw., New York, NY, USA, 2014, pp. 447-458.

[74] Z. Li, M. Jiang, X. Zhang, X. Chen, and W. Hou, "Space-time-multiplexed multiimage visible light positioning system exploiting Pseudo-Miller-coding for smart phones," IEEE Trans. Wireless Commun., vol. 16, no. 12, pp. 8261-8274, Dec. 2017.

[75] M. S. Rahman and K.-D. Kim, "Indoor location estimation using visible light communication and image sensors," Int. J. Smart Home, vol. 7, no. 1, pp. 99-114, 2013.

[76] R. Zhang, W.-D. Zhong, K. Qian, and D. Wu, "Image sensor based visible light positioning system with improved positioning algorithm," IEEE Access, vol. 5, pp. 6087-6094, 2017

[77] L. C. Png, L. Chen, S. Liu, and W. K. Peh, "An arduino-based indoor positioning system (IPS) using visible light communication and ultrasound," in Proc. IEEE Int. Conf. Consum. Electron.-Taiwan, May 2014, pp. 217-218.

[78] K. Dividis, "Design and prototyping of a visible light indoor positioning system." Stan Ackermans Instituut, 2007.

[79] C. Zhang and X. Zhang, "LiTell: Robust indoor localization using unmodified light fixtures," in Proc. 22nd Annu. Int. Conf. Mobile Comput. Netw., 2016, pp. 230-242.

[80] C. Zhang, S. Zhou, and X. Zhang, "Visible light localization using incumbent light fixtures: Demo abstract," in Proc. 14th ACM Conf. Embedded Netw. Sensor Syst., 2016, pp. 304-305.

[81] S. Gezici and H. V. Poor, "Position estimation via ultra-wide-band signals," Proc. IEEE, vol. 97, no. 2, pp. 386-403, Feb. 2009.

[82] A. J. Weiss, "Direct position determination of narrowband radio frequency transmitters," IEEE Signal Process. Lett., vol. 11, no. 5, pp. 513-516, May 2004.

[83] O. Bialer, D. Raphaeli, and A. J. Weiss, "Maximum-likelihood direct position estimation in dense multipath," IEEE Trans. Veh. Technol., vol. 62, no. 5, pp. 2069-2079, Jun. 2013.

[84] N. Garcia, H. Wymeersch, E. G. Larsson, A. M. Haimovich, and M. Coulon, "Direct localization for massive MIMO," IEEE Trans. 
Signal Process., vol. 65, no. 10, pp. 24752487, May 2017.

[85] A. J. Weiss and A. Amar, "Direct position determination of multiple radio signals," EURASIP J. Adv. Signal Process., vol. 2005, no. 1, p. 653549, 2005.

[86] A. Amar and A. J. Weiss, "Localization of narrowband radio emitters based on Doppler frequency shifts," IEEE Trans. Signal Process., vol. 56, no. 11, pp. 5500-5508, Nov. 2008.

[87] N. Vankayalapati, S. Kay, and Q. Ding, "TDOA based direct positioning maximum likelihood estimator and the Cramer-Rao bound," IEEE Trans. Aerosp. Electron. Syst., vol. 50, no. 3, pp. 1616-1635, Jul. 2014.

[88] L. Tzafri and A. J. Weiss, "High-resolution direct position determination using MVDR," IEEE Trans. Wireless Commun., vol. 15, no. 9, pp. 6449-6461, Sep. 2016.

[89] G. Kail, P. Maechler, N. Preyss, and A. Burg, "Robust asynchronous indoor localization using LED lighting," in Proc. IEEE Int. Conf. Acoust. Speech Signal Process. (ICASSP), May 2014, pp. 1866-1870.

[90] A. Goldsmith, Wireless Communications. Cambridge, U.K.: Cambridge Univ. Press, 2005.

[91] S. De Lausnay, L. De Strycker, J.-P. Goemaere, B. Nauwelaers, and N. Stevens, "A survey on multiple access visible light positioning," in Proc. IEEE Int. Conf. Emerg. Technol. Innov. Bus. Pract. Transformation Soc. (EmergiTech), Aug. 2016, pp. 38-42.

[92] Y. Wang, S. Ma, and C. L. P. Chen, "TOAbased passive localization in quasisynchronous networks," IEEE Commun. Lett., vol. 18, no. 4, pp. 592-595, Apr. 2014.

[93] J. M. Kahn and J. R. Barry, "Wireless infrared communications," Proc. IEEE, vol. 85, no. 2, pp. 265-298, Feb. 1997.

[94] E. Kazikli and S. Gezici (2018). "Hybrid TDOA/RSS based localization for visible light systems.” [Online]. Available: https:// arxiv.org/abs/1802.03991

[95] N. Patwari, J. N. Ash, S. Kyperountas, A. O. Hero, R. L. Moses, and N. S. Correal, "Locating the nodes: Cooperative localization in wireless sensor networks," IEEE Signal Process. Mag., vol. 22, no. 4, pp. 54-69, Jul. 2005.

[96] H. L. Van Trees, Detection, Estimation, and Modulation Theory. New York, NY, USA: Wiley, 2004.

[97] Y. Qi, H. Kobayashi, and H. Suda, "Analysis of wireless geolocation in a non-line-ofsight environment," IEEE Trans. Wireless Commun., vol. 5, no. 3, pp. 672-681, Mar. 2006.

[98] C. G. Gavrincea, J. Baranda, and P. Henarejos, "Rapid prototyping of standard-compliant visible light communications system," IEEE Commun. Mag., vol. 52, no. 7, pp. 80-87, Jul. 2014.

[99] W. Gu, M. Aminikashani, P. Deng, and M. Kavehrad, "Impact of multipath reflections on the performance of indoor visible light positioning systems," J. Lightw. Technol., vol. 34, no. 10, pp. 2578-2587, May 15, 2016.

[100] S.-H. Yang, E.-M. Jung, and S.-K. Han, "Indoor location estimation based on LED visible light communication using multiple optical receivers," IEEE Commun. Lett., vol. 17, no. 9, pp. 1834-1837, Sep. 2013.
[101] X. Zhang, J. Duan, Y. Fu, and A. Shi, "Theoretical accuracy analysis of indoor visible light communication positioning system based on received signal strength indicator," J. Lightw. Technol., vol. 32, no. 21, pp. 4180-4186, Nov. 1, 2014.

[102] H. Steendam, T. Q. Wang, and J. Armstrong, "Theoretical lower bound for indoor visible light positioning using received signal strength measurements and an aperture-based receiver," J. Lightw. Technol., vol. 35, no. 2, pp. 309-319, Jan. 15, 2017.

[103] E. Gonendik and S. Gezici, "Fundamental limits on RSS based range estimation in visible light positioning systems," IEEE Commun. Lett., vol. 19, no. 12, pp. 2138-2141, Dec. 2015.

[104] M. Aminikashani, W. Gu, and M. Kavehrad, "Indoor location estimation with optical-based orthogonal frequency division multiplexing communications," Opt. Eng., vol. 55, no. 5, p. 056116, 2016.

[105] G. Turin, "An introduction to matched filters," IRE Trans. Inf. Theory, vol. 6, no. 3, pp. 311-329, Jun. 1960.

[106] J. Luo, L. Fan, and H. Li, "Indoor positioning systems based on visible light communication: State of the art," IEEE Commun. Surveys Tuts., vol. 19, no. 4, pp. 2871-2893, 4th Quart., 2017.

[107] J. H. Y. Nah, R. Parthiban, and M. H. Jaward, "Visible light communications localization using TDOAbased coherent heterodyne detection," in Proc. IEEE 4th Int. Conf. Photon. (ICP), Oct. 2013, pp. 247-249.

[108] K. Panta and J. Armstrong, "Indoor localisation using white LEDs," Electron. Lett., vol. 48, no. 4, pp. 228-230, 2012.

[109] A. Arafa, X. Jin, and R. Klukas, "Wireless indoor optical positioning with differential photosensor," IEEE Photon. Technol. Lett., vol. 24, no. 12, pp. 1027-1029, Jun. 15, 2012.

[110] D. Zheng, G. Chen, and J. A. Farrell, "Navigation using linear photo detector arrays," in Proc. IEEE Int. Conf. Control Appl. (CCA), Aug. 2013, pp. 533-538.

[111] H. Steendam, T. Q. Wang, and J. Armstrong, "Cramer-Rao bound for AOA-based VLP with an aperture-based receiver," in Proc. IEEE Int. Conf. Commun. (ICC), May 2017, pp. 1-6.

[112] S. H. Yang, E. M. Jeong, and S. K. Han, "Indoor positioning based on received optical power difference by angle of arrival," Electron. Lett., vol. 50, no. 1 , pp. 49-51, Jan. 2014.

[113] M. Yasir, S.-W. Ho, and B. N. Vellambi, "Indoor position tracking using multiple optical receivers," J. Lightw. Technol., vol. 34, no. 4, pp. 1166-1176, Feb. 15, 2016.

[114] S.-H. Yang, H.-S. Kim, Y.-H. Son, and S.-K. Han, "Three-dimensional visible light indoor localization using AOA and RSS with multiple optical receivers," J. Lightw. Technol., vol. 32, no. 14, pp. 2480-2485, Jul. 15, 2014.

[115] A. Arafa, S. Dalmiya, R. Klukas, and J. F. Holzman, "Angle-of-arrival reception for optical wireless location technology," Opt. Exp., vol. 23, no. 6, pp. 7755-7766, 2015.

[116] P. Cherntanomwong and W. Chantharasena, "Indoor localization system using visible light communication," in Proc. 7th Int. Conf. Inf. Technol. Elect. Eng. (ICITEE), Oct. 2015, pp. 480-483.
[117] C. Sertthin, E. Tsuji, M. Nakagawa, S. Kuwano, and K. Watanabe, "A switching estimated receiver position scheme for visible light based indoor positioning system," in Proc. 4th Int. Symp. Wireless Pervas. Comput., Feb. 2009, pp. 1-5.

[118] M. T. Taylor and S. Hranilovic, "Angular diversity approach to indoor positioning using visible light," in Proc. IEEE Globecom Workshops, Dec. 2013, pp. 1093-1098.

[119] Y. H. Choi, I. H. Park, Y. H. Kim, and J. Y. Kim, "Novel LBS technique based on visible light communications," in Proc. IEEE Int. Conf. Consum. Electron. (ICCE), Jan. 2012, pp. 576-577.

[120] X. Guo, S. Shao, N. Ansari, and A. Khreishah, "Indoor localization using visible light via fusion of multiple classifiers," IEEE Photon. J., vol. 9, no. 6, Dec. 2017, Art. no. 7803716.

[121] A. M. Vegni and M. Biagi, "An indoor localization algorithm in a small-cell LEDbased lighting system," in Proc. Int. Conf. Indoor Positioning Indoor Navigat. (IPIN), Nov. 2012, pp. 1-7.

[122] M. Biagi, A. M. Vegni, and T. D. C. Little, "LAT indoor MIMO-VLC-Localize, access and transmit," in Proc. Int. Workshop Opt. Wireless Commun. (IWOW), Oct. 2012, pp. 1-3.

[123] Q. Liang and M. Liu (2017). "Plugo: A VLC systematic perspective of large-scale indoor localization." [Online]. Available: https:// arxiv.org/abs/1709.06926

[124] Y. U. Lee and G. Jung, "Secure positioning technique based on the encrypted visible light map," Proc. SPIE, vol. 10128, p. $101280 \mathrm{~K}$, Jan. 2017.

[125] M. Liu et al., "Towards indoor localization using visible light communication for consumer electronic devices," in Proc. IEEE/RSJ Int. Conf. Intell. Robot. Syst., Sep. 2014, pp. 143-148.

[126] Y.-Y. Won, S.-H. Yang, D.-H. Kim, and S.-K. Han, "Three-dimensional optical wireless indoor positioning system using location code map based on power distribution of visible light emitting diode," IET Optoelectron., vol. 7, no. 3, pp. 77-83, Jun. 2013.

[127] S.-H. Yang, D.-R. Kim, H.-S. Kim, Y.-H. Son, and S.-K. Han, "Visible light based high accuracy indoor localization using the extinction ratio distributions of light signals," Microw. Opt. Technol. Lett., vol. 55, no. 6, pp. 1385-1389, 2013.

[128] A. Conti, M. Guerra, D. Dardari, N. Decarli, and M. Z. Win, "Network experimentation for cooperative localization," IEEE J. Sel. Areas Commun., vol. 30, no. 2, pp. 467-475, Feb. 2012.

[129] D. Dardari, A. Conti, J. Lien, and M. Z. Win, "The effect of cooperation on UWB-based positioning systems using experimental data," EURASIP J. Adv. Signal Process., vol. 2008, p. 513873, Dec. 2008.

[130] B. Teague, Z. Liu, F. Meyer, and M. Z. Win, "Peregrine: 3-D network localization and navigation," in Proc. IEEE 9th Latin-Amer. Conf. Commun. (LATINCOM), Nov. 2017, pp. 1-6.

[131] Z. Liu, W. Dai, and M. Z. Win, "Mercury: An infrastructure-free system for network localization and navigation," IEEE Trans. Mobile Comput., vol. 17, no. 5 , pp. 1119-1133, May 2018.

[132] U. A. Khan, S. Kar, and J. M. F. Moura, "DILAND: An algorithm for distributed 
sensor localization with noisy distance measurements," IEEE Trans. Signal Process. vol. 58, no. 3, pp. 1940-1947, Mar. 2010

[133] F. Meyer, O. Hlinka, and F. Hlawatsch "Sigma point belief propagation," IEEE Signal Process. Lett., vol. 21, no. 2, pp. 145-149, Feb. 2014.

[134] M. Yasir, S.-W. Ho, and B. N. Vellambi, "Indoor positioning system using visible light and accelerometer," J. Lightw. Technol., vol. 32, no. 19, pp. 3306-3316, Oct. 1, 2014.

[135] D. Butnariu, Y. Censor, P. Gurfil, and E. Hadar, "On the behavior of subgradient projections methods for convex feasibility problems in Euclidean spaces," SIAM J. Optim., vol. 19, no. 2, pp. 786-807, 2008.

[136] S. Yousefi, H. Wymeersch, X.-W. Chang, and B. Champagne, "Tight twodimensional outer-approximations of feasible sets in wireless sensor networks," IEEE Commun. Lett., vol. 20, no. 3, pp. 570-573, Mar. 2016.

[137] S. Yousefi, X.-W. Chang, and B. Champagne, "Mobile localization in non-line-of-sight using constrained squareroot unscented Kalman filter," IEEE Trans. Veh. Technol., vol. 64, no. 5, pp. 2071-2083, May 2015.

[138] H. H. Bauschke and J. M. Borwein, "On projection algorithms for solving convex feasibility problems," SIAM Rev., vol. 38 no. 3, pp. 367-426, Sep. 1996.

[139] Y. Censor and A. Segal, "Algorithms for the quasiconvex feasibility problem," J. Comput. Appl. Math., vol. 185, no. 1, pp. 34-50, 2006.

[140] Y. Censor and A. Lent, "Cyclic subgradient projections," Math. Program., vol. 24, no. 1 , pp. 233-235, 1982.

[141] P. L. Combettes, "Quasi-Fejérian analysis of some optimization algorithms," in Inherently Parallel Algorithms in Feasibility and Optimization and their Applications (Studies in Computational Mathematics), vol. 8, D. Butnariu, S. Reich, and Y. Censor, Eds. Amsterdam, The Netherlands: Elsevier, 2001, pp. 115-152.

[142] S. Boyd, A. Ghosh, B. Prabhakar, and D. Shah, "Randomized gossip algorithms," IEEE/ACM Trans. Netw., vol. 52, no. 6, pp. 2508-2530, Jun. 2006.

[143] H. Ma, L. Lampe, and S. Hranilovic, "Coordinated broadcasting for multiuser indoor visible light communication systems," IEEE Trans. Commun., vol. 63, no. 9, pp. 3313-3324, Sep. 2015.

[144] Z. Zhou, M. Kavehrad, and P. Deng, "Indoor positioning algorithm using lightemitting diode visible light

communications," Opt. Eng., vol. 51, no. 8, p. 085009, 2012.

[145] W. W.-L. Li, Y. Shen, Y. J. Zhang, and M. Z. Win, "Robust power allocation for energy-efficient location-aware networks," IEEE/ACM Trans. Netw., vol. 21, no. 6, pp. 1918-1930, Dec. 2013.

[146] Y. Shen, W. Dai, and M. Z. Win, "Power optimization for network localization," IEEE/ACM Trans. Netw., vol. 22, no. 4, pp. 1337-1350, Aug. 2014.

[147] W. Dai, Y. Shen, and M. Z. Win, "Distributed power allocation for cooperative wireless network localization," IEEE J. Sel. Areas Commun., vol. 33, no. 1, pp. 28-40, Jan. 2015.

[148] J. Chen, W. Dai, Y. Shen, V. K. N. Lau, and M. Z. Win, "Power management for cooperative localization: A game theoretical approach," IEEE Trans. Signal Process., vol. 64, no. 24, pp. 6517-6532, Dec. 2016.

[149] W. Dai, Y. Shen, and M. Z. Win, "A computational geometry framework for efficient network localization," IEEE Trans. Inf. Theory, vol. 64, no. 2, pp. 1317-1339, Feb. 2018.

[150] A. Shahmansoori, G. Seco-Granados, and H. Wymeersch, "Power allocation for
OFDM wireless network localization under expectation and robustness constraints," IEEE Trans. Wireless Commun., vol. 16, no. 3, pp. 2027-2038, Mar. 2017.

[151] L. Chen, B. Krongold, and J. Evans, "Theoretical characterization of nonlinear clipping effects in IM/DD optical OFDM systems," IEEE Trans. Commun., vol. 60, no. 8, pp. 2304-2312, Aug. 2012.

[152] X. Ling, J. Wang, X. Liang, Z. Ding, and C. Zhao, "Offset and power optimization for DCO-OFDM in visible light communication systems," IEEE Trans. Signal Process., vol. 64, no. 2, pp. 349-363, Jan. 2016

[153] Y. S. Eroglu, A. Sahin, I. Güvenç, N. Pala, and M. Yüksel, "Multi-element transmitter design and performance evaluation for visible light communication," in Proc. IEEE Globecom Workshops (GC Wkshps), Dec. 2015, pp. 1-6.

[154] E. F. Schubert, Light-Emitting Diodes. Cambridge, U.K.: Cambridge Univ. Press, 2003.

[155] A. Pandharipande and D. Caicedo, "Adaptive illumination rendering in LED lighting systems," IEEE Trans. Syst. Man Cybern., Syst., vol. 43, no. 5, pp. 1052-1062, Sep. 2013.

[156] S. Boyd and L. Vandenberghe, Convex Optimization. Cambridge, U.K.: Cambridge Univ. Press, 2004

[157] E. Xu, Z. Ding, and S. Dasgupta, "Source localization in wireless sensor networks from signal time-of-arrival measurements," IEEE Trans. Signal Process., vol. 59, no. 6, pp. 2887-2897, Jun. 2011.

[158] A. Mostafa and L. Lampe, "Optimal and robust beamforming for secure transmission in MISO visible-light communication links," IEEE Trans. Signal Process., vol. 64, no. 24, pp. 6501-6516, Dec. 2016.

\section{ABOUT THE AUTHORS}

Musa Furkan Keskin received the B.S. and M.S. degrees from the Department of Electrical and Electronics Engineering, Bilkent University, Ankara, Turkey, in 2010 and 2012, respectively. Currently, he is working towards the Ph.D. degree in the same department. His research interests lie in the fields of wireless localization, signal processing, and visible light communications.

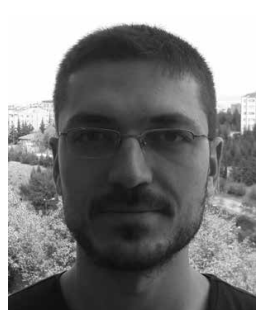

Ahmet Dundar Sezer was born in 1989 in Emet, Kutahya, Turkey. He received the B.S. and M.S. degrees in electrical and electronics engineering from Bilkent University, Ankara, Turkey, in 2011 and 2013, respectively, where he is currently working toward the Ph.D. degree.

His current research interests include signal processing, wireless communications, and optimization.

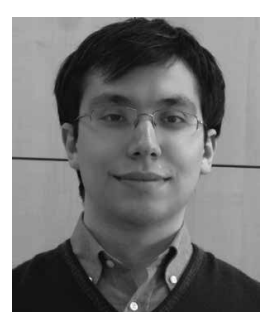

Sinan Gezici (Senior Member, IEEE) received the B.S. degree from Bilkent University, Ankara, Turkey, in 2001 and the Ph.D. degree in electrical engineering from Princeton University, Princeton, NJ, USA, in 2006.

From 2006 to 2007, he worked at Mitsubishi Electric Research Laboratories, Cambridge, MA, USA. Since 2007, he has been with the Department of Electrical and Electronics Engineering,

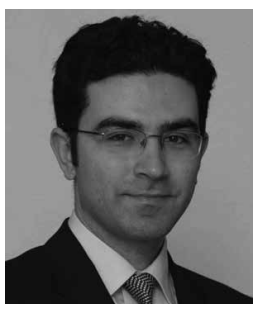
Bilkent University, where he is currently a Professor. His research interests are in the areas of detection and estimation theory, wireless communications, and localization systems. Among his publications in these areas is the book Ultra-Wideband Positioning Systems: Theoretical Limits, Ranging Algorithms, and Protocols (Cambridge, U.K.: Cambridge Univ. Press, 2008).

Dr. Gezici was an Associate Editor for the IEEE Transactions on Communications, IEEE Wireless Communications Letters, and Journal of Communications and Networks. 